\title{
Location invariant Weiss-Hill estimator
}

\author{
Chengxiu Ling · Zuoxiang Peng • Saralees Nadarajah
}

Received: 13 January 2010 / Revised: 21 April 2011 /

Accepted: 12 May 2011 / Published online: 4 June 2011

(C) Springer Science+Business Media, LLC 2011

\begin{abstract}
In this paper, a new kind of location invariant Weiss-Hill estimator of the extreme value index $\gamma \in \mathbb{R}$ is proposed. The new estimator is a combination of two estimators proposed by Weiss (Nav Res Logist Q 1:111-114, 1971) and Fraga Alves (Extremes 4:199-217, 2001a). The following properties of the new estimator are derived: weak consistency, strong consistency, and asymptotic expansions. A bias corrected version of the proposed estimator is given after determining an optimal sample fraction. Some finite simulation studies are performed.
\end{abstract}

Keywords Extreme value index · Location invariant - Mean squared error · Second order regular variation

AMS 2000 Subject Classifications Primary-62G32; Secondary-65C05

\section{Introduction}

Let $\left\{X_{n}, n \geq 1\right\}$ be an independent and identically distributed random variable sequence with common distribution function (d.f) $F(x)$. Let $X_{1, n} \leq X_{2, n} \leq \cdots \leq$

\section{Ling}

Department of Actuarial Science, Faculty of Business and Economics, University of Lausanne, Bâtiment Extranef, UNIL-Dorigny, 1015 Lausanne, Switzerland

\section{Z. Peng}

School of Mathematics and Statistics, Southwest University, Chongqing, 400715, China

S. Nadarajah $(\bowtie)$

School of Mathematics, University of Manchester, Manchester, UK

e-mail: mbbsssn2@manchester.ac.uk 
$X_{n, n}$ denote the associated increasing order statistics of $X_{1}, X_{2}, \ldots, X_{n}$. Suppose that $F \in D\left(G_{\gamma}\right)$, i.e. there exist real numbers $a_{n}>0$ and $b_{n} \in \mathbb{R}$ such that

$$
P\left(\frac{X_{n, n}-b_{n}}{a_{n}} \leq x\right)=F^{n}\left(a_{n} x+b_{n}\right) \stackrel{w}{\rightarrow} G_{\gamma}(x)
$$

as $n \rightarrow \infty$, where

$$
G_{\gamma}(x)=\left\{\begin{array}{lll}
\exp \left\{-(1+\gamma x)^{-1 / \gamma}\right\}, & \text { for } 1+\gamma x>0, & \text { if } \gamma \neq 0, \\
\exp \{-\exp (-x)\}, & \text { for } x \in \mathbb{R}, & \text { if } \gamma=0 .
\end{array}\right.
$$

Note that $\gamma$ is referred to the extreme value index.

A challenge in theoretical and empirical analysis of extreme value theory is how to estimate the extreme value index if the marginal d.f $F$ is unknown. Hill (1975) proposed the Hill-type estimator for $\gamma>0$, i.e.

$$
\widehat{\gamma}_{n}^{H}(k)=\frac{1}{k} \sum_{i=0}^{k-1} \log X_{n-i, n}-\log X_{n-k, n} .
$$

The asymptotic properties of the Hill estimator have been studied by de Haan (1994). Dekkers et al. (1989) extended the Hill estimator to the moment-type estimator given by

$$
\widehat{\gamma}_{n}^{M}(k)=\widehat{\gamma}_{n}^{+}(k)+\widehat{\gamma}_{n}^{-}(k)
$$

for $\gamma \in \mathbb{R}$, where $\widehat{\gamma}_{n}^{+}(k)$ is given by Eq. 1.1 and

$$
\widehat{\gamma}_{n}^{-}(k)=1-\frac{1}{2}\left\{1-\frac{\left(M_{n}^{(1)}(k)\right)^{2}}{M_{n}^{(2)}(k)}\right\}^{-1},
$$

where

$$
M_{n}^{(j)}(k)=\frac{1}{k} \sum_{i=0}^{k-1}\left(\log \frac{X_{n-i, n}}{X_{n-k, n}}\right)^{j}
$$

for $j=1$, 2. Fraga Alves et al. (2009) considered another estimator, named as the mixed moment estimator, and defined for $\gamma \in \mathbb{R}$ as

$$
\widehat{\gamma}_{n}^{\text {mix }}{ }^{\text {M }}(k)=\frac{\widehat{\varphi}_{n}(k)-1}{1+2 \min \left(\widehat{\varphi}_{n}(k)-1,0\right)}
$$

with

$$
\widehat{\varphi}_{n}(k):=\frac{M_{n}^{(1)}(k)-L_{n}^{(1)}(k)}{\left(L_{n}^{(1)}(k)\right)^{2}}, \quad L_{n}^{(1)}(k):=\frac{1}{k} \sum_{i=0}^{k-1}\left(1-\frac{X_{n-k, n}}{X_{n-i, n}}\right) .
$$


The corrected Hill estimator considered is the one introduced in Caeiro et al. (2005) and given by

$$
\widehat{\gamma}_{n}^{C H}(k)=\widehat{\gamma}_{n}^{+}(k)\left(1-\frac{\widehat{\beta}}{1-\widehat{\rho}}(n / k)^{\widehat{\rho}}\right),
$$

where $(\widehat{\beta}, \widehat{\rho})$ are joint external estimators of the "scale" and "shape" parameters, $(\beta, \rho)$, in the $A(t)$ function defined by Eq. 1.12 in Caeiro et al. (2005). For convenient estimation of $(\beta, \rho)$, see algorithms in Gomes and Pestana (2007) and in Gomes et al. (2008b) for the corrected-moment type estimator (denoted by $\left.\widehat{\gamma}_{n}^{C M}(k)\right)$ :

$$
\widehat{\gamma}_{n}^{C M}(k)=\widehat{\gamma}_{n}^{M}(k)\left(1-\frac{\widehat{\beta}}{1-\widehat{\rho}}\left(\frac{n}{k}\right)^{\widehat{\rho}}\right)-\frac{\widehat{\beta} \widehat{\rho}}{(1-\widehat{\rho})^{2}}\left(\frac{n}{k}\right)^{\widehat{\rho}} .
$$

Falk (1995) proposed a Hill-type estimator for $\gamma<0$ :

$$
\widehat{\gamma}_{n}^{N H}(k)=\frac{1}{k-1} \sum_{i=2}^{k} \log \frac{X_{n, n}-X_{n-i+1, n}}{X_{n, n}-X_{n-k, n}} .
$$

Müller and Hüsler (2005) improved the above estimator by providing an "iterative estimator":

$$
\widehat{\gamma}_{n}^{I H}(q)=\widehat{\gamma}_{n}^{I H}(q, k)=\frac{1}{k-1} \sum_{i=2}^{k} \log \frac{\widehat{w}-X_{n-i+1, n}}{\widehat{w}-X_{n-k, n}}
$$

where

$$
\widehat{w}=\sum_{j=1}^{q} a_{j}\left(\widehat{\gamma}_{1}\right) X_{n-j+1, n}
$$

where $\widehat{\gamma}_{1}$ is any consistent estimator of $\gamma$, the weights $\mathbf{a}=\left(a_{1}, a_{2}, \ldots, a_{q}\right)^{T}$ are given by

$$
\mathbf{a}=\frac{\boldsymbol{\Lambda}^{-1}\left[\left(\boldsymbol{v}^{T} \boldsymbol{\Lambda}^{-1} \boldsymbol{v}\right) \mathbf{1}_{q}-\left(\mathbf{1}_{q}^{T} \boldsymbol{\Lambda}^{-1} \boldsymbol{v}\right) \boldsymbol{v}\right]}{\left(\boldsymbol{v}^{T} \boldsymbol{\Lambda}^{-1} \boldsymbol{v}\right)\left(\mathbf{1}_{q}^{T} \boldsymbol{\Lambda}^{-1} \mathbf{1}_{q}\right)-\left(\mathbf{1}_{q}^{T} \boldsymbol{\Lambda}^{-1} \boldsymbol{v}\right)^{2}}
$$

with $\boldsymbol{\Lambda}=\lambda_{i j}$ the symmetric $q \times q$ matrix given by

$$
\lambda_{i j}=\frac{\Gamma\left(-2 \widehat{\gamma_{1}}+i\right) \Gamma\left(-\widehat{\gamma}_{1}+j\right)}{\Gamma\left(-\widehat{\gamma}_{1}+i\right) \Gamma(j)}, j \leq i,
$$




$$
\boldsymbol{v}=\left(\frac{\Gamma\left(-\widehat{\gamma}_{1}+1\right)}{\Gamma(1)}, \frac{\Gamma\left(-\widehat{\gamma_{1}}+2\right)}{\Gamma(2)}, \ldots, \frac{\Gamma\left(-\widehat{\gamma}_{1}+q\right)}{\Gamma(q)}\right)^{T},
$$

and with $\mathbf{1}_{k}$ denoting a $k$ vector of ones. For details, see Müller and Hüsler (2005, page 141).

For general $\gamma \in \mathbb{R}$, Pickands (1975) proposed the following estimator:

$$
\widehat{\gamma}_{n}^{P}(m)=\frac{1}{\log 2} \log \frac{X_{n-m+1, n}-X_{n-2 m+1}}{X_{n-2 m+1, n}-X_{n-4 m+1}} .
$$

For asymptotic properties of the Pickands estimator, see Dekkers and de Haan (1989). For some extensions of the Pickands estimator, see Peng (1997) and Segers (2005). For $\gamma>0$, we can use the following simplified Pickands estimator

$$
\widehat{\gamma}_{n}^{S P}(m, d)=\frac{1}{\log d} \log \frac{X_{n-m+1, n}}{X_{n-k+1, n}},
$$

where $k=k(n)$ and $m=m(n)$ are integers such that $\min (k, m) \rightarrow \infty$, $\max (k, m) / n \rightarrow 0$ and $k / m \rightarrow d \neq 1$ as $n \rightarrow \infty$. For more details, see Peng (1998b). Weiss (1971) and Peng and Nadarajah (2001) studied the following Weiss-type Pickands estimator

$$
\widehat{\gamma}_{n}^{W}(m)=\frac{1}{\log 2} \log \frac{X_{n, n}-X_{n-m+1, n}}{X_{n, n}-X_{n-2 m+1, n}}
$$

for $\gamma<0$, where $m=m(n)$ is an intermediate integer, i.e. $m(n) \rightarrow \infty$ and $m(n) / n \rightarrow 0$ as $n \rightarrow \infty$. For some extensions of Eq. 1.8, see He (2004). For $\gamma \in \mathbb{R}$, Fraga Alves (2001b) studied the combined Weiss-Hill estimator defined by

$$
\widehat{\gamma}_{n}^{W H}(k)=\widehat{\gamma}_{n}^{H}(k)+\widehat{\gamma}_{n}^{W}(m)
$$

with $m=[(k+1) / 2]$ in Weiss estimator. Araújo Santos et al. (2006) proposed several location invariant estimators, called Peaks Over Random Threshold estimators (PORT) (denoted by $\left.\widehat{\gamma}_{n}^{P H}(p), \widehat{\gamma}_{n}^{P M}(p), \widehat{\gamma}_{n}^{M M}(p)\right)$, based on Eqs. 1.1, 1.2, 1.3, i.e.

$$
\begin{aligned}
& \widehat{\gamma}_{n}^{P H}(p)=\widehat{\gamma}_{n}^{P H}(p, k)=\widehat{\gamma}_{n}^{H}\left(k, X_{i}^{*}, 1 \leq i \leq n\right), \\
& \widehat{\gamma}_{n}^{P M}(p)=\widehat{\gamma}_{n}^{P M}(p, k)=\widehat{\gamma}_{n}^{M}\left(k, X_{i}^{*}, 1 \leq i \leq n\right)
\end{aligned}
$$

and

$$
\widehat{\gamma}_{n}^{M M}(p)=\widehat{\gamma}_{n}^{M M}(p, k)=\widehat{\gamma}_{n}^{\operatorname{mix} M}\left(k, X_{i}^{*}, 1 \leq i \leq n\right),
$$


where

$$
X_{i}^{*}:=X_{i}-X_{[n p]+1, n}, \quad 0 \leq p<1,1 \leq i \leq n .
$$

Note that the estimators given by Eqs. 1.7 and 1.8 are location invariant. But the estimators given by Eqs. 1.1, 1.2, 1.3 and 1.9 are not location invariant.

Fraga Alves (2001a) also proposed the following location invariant Hill-type estimator:

$$
\widehat{\gamma}_{n}^{H}\left(k_{0}, k\right)=\frac{1}{k_{0}} \sum_{i=0}^{k_{0}-1} \log \frac{X_{n-i, n}-X_{n-k, n}}{X_{n-k_{0}, n}-X_{n-k, n}},
$$

where $k_{0}=k_{0}(k) \rightarrow \infty, k=k(n) \rightarrow \infty, k_{0} / k \rightarrow 0$ and $k / n \rightarrow 0$ as $n \rightarrow$ $\infty$. The asymptotic properties like weak consistency and asymptotic distributions were considered by Fraga Alves (2001a) under some second order regular varying conditions. Ling et al. (2007a, b, 2009) extended Eq. 1.13 to the following location invariant moment-type estimator for general $\gamma \in \mathbb{R}$ :

$$
\widehat{\gamma}_{n}^{\text {Invm }}\left(k_{0}, k\right)=\widehat{\gamma}_{n}^{+}\left(k_{0}, k\right)+\widehat{\gamma}_{n}^{-}\left(k_{0}, k\right),
$$

where $\widehat{\gamma}_{n}^{+}\left(k_{0}, k\right)$ is given by Eq. 1.13 and

$$
\widehat{\gamma}_{n}^{-}\left(k_{0}, k\right)=1-\frac{1}{2}\left\{1-\frac{\left(M_{n}^{(1)}\left(k_{0}, k\right)\right)^{2}}{M_{n}^{(2)}\left(k_{0}, k\right)}\right\}^{-1},
$$

where

$$
M_{n}^{(j)}\left(k_{0}, k\right)=\frac{1}{k_{0}} \sum_{i=0}^{k_{0}-1}\left(\log \frac{X_{n-i, n}-X_{n-k, n}}{X_{n-k_{0}, n}-X_{n-k, n}}\right)^{j}
$$

for $j=1,2$. For more related work on extreme value index estimation, see Beirlant et al. (1996), de Haan and Peng (1998), Peng (1998a), Gomes and Martins (2002, 2004), Peng and Qi (2006a, b), Gomes et al. (2008a, b), Gomes and Henriques Rodrigues (2008), Li et al. (2008, 2010) and Qi (2010).

In this paper, we study the following combined Weiss-Hill estimator for $\gamma \in \mathbb{R}$ :

$$
\widehat{\gamma}_{n}^{\text {Invwh }}\left(k_{0}, k\right)=\widehat{\gamma}_{n}^{W}(m)+\widehat{\gamma}_{n}^{H}\left(k_{0}, k\right),
$$

where $\widehat{\gamma}_{n}^{W}(m)$ and $\widehat{\gamma}_{n}^{H}\left(k_{0}, k\right)$ are given by Eqs. 1.8 and 1.13, respectively, and $m=\left[\left(k_{0}+1\right) / 2\right], k_{0}=k_{0}(k) \rightarrow \infty, \quad k=k(n) \rightarrow \infty, \quad k_{0} / k \rightarrow 0, \quad k / n \rightarrow 0$

as $n \rightarrow \infty$. Obviously, the estimator given by Eq. 1.15 is location invariant. 
The contents of this paper are organized as follows. In Section 2, the weak and strong consistencies of the location invariant estimator $\widehat{\gamma}_{n}^{\text {Invwh }}\left(k_{0}, k\right)$ are derived as $F \in D\left(G_{\gamma}\right)$ (Theorems 2.1 and 2.2). The asymptotical expansions of $\widehat{\gamma}_{n}^{\text {Invwh }}\left(k_{0}, k\right)$ are considered under some second order regular varying conditions (Theorems 2.3 and 2.4). The optimal choice of the sample fraction, $k_{0}$, by the criterion of minimizing the asymptotic mean squared error, is discussed (Theorem 2.5). Simulation studies are performed in Section 3. All proofs are given in Section 4.

\section{Asymptotic properties}

In what follows we assume that $F \in D\left(G_{\gamma}\right)$ for $\gamma \in \mathbb{R}$, or equivalently suppose that for all $x>0$

$$
\frac{U(t x)-U(t)}{a(t)} \rightarrow D_{\gamma}(x):= \begin{cases}\frac{x^{\gamma}-1}{\gamma}, & \gamma \neq 0 \\ \log x, & \gamma=0\end{cases}
$$

as $t \rightarrow \infty$, where $U$ denotes the inverse function of $1 /(1-F)$ and the auxiliary function $a(t)$ is a regular varying function with index $\gamma$. In fact,

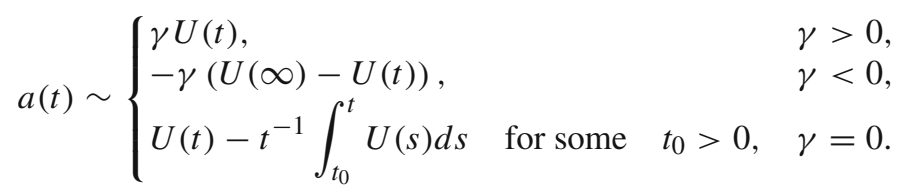

Firstly, we establish weak and strong consistencies of the proposed estimator $\widehat{\gamma}_{n}^{\text {Invwh }}\left(k_{0}, k\right)$.

Theorem 2.1 Suppose that $F \in D\left(G_{\gamma}\right)$ and Eq. 1.16 hold. Then $\widehat{\gamma}_{n}^{\text {Invwh }}\left(k_{0}, k\right)$ converges to $\gamma$ in probability.

Theorem 2.2 Assume that Eq.2.1 holds for $\gamma \in \mathbb{R}$. Additionally, suppose that integers $k_{0}, k$ and $m$ satisfy Eq. 1.16 and $k_{0} /(\log k)^{\delta_{1}} \rightarrow \infty, k /(\log n)^{\delta_{2}} \rightarrow \infty$ as $n \rightarrow \infty$ for some $\delta_{1}>0$ and $\delta_{2}>0$. Then $\widehat{\gamma}_{n}^{\text {Invwh }}\left(k_{0}, k\right)$ converges to $\gamma$ almost surely.

In order to obtain the asymptotic distribution of $\widehat{\gamma}_{n}^{\text {Invwh }}\left(k_{0}, k\right)$, we consider the following second order regular varying condition:

$$
\lim _{t \rightarrow \infty} \frac{\frac{U(t x)-U(t)}{a(t)}-D_{\gamma}(x)}{A(t)}=k_{\gamma, \rho}(x)
$$


for all $x>0$, where

$$
k_{\gamma, \rho}(x)= \begin{cases}(\log x)^{2} / 2, & \text { if } \rho=\gamma=0, \\ \left(x^{\gamma} \log x\right) / \gamma, & \text { if } \rho=0, \gamma \neq 0, \\ \log x, & \text { if } \rho<0, \gamma+\rho=0, \\ \left(x^{\gamma+\rho}-1\right) /(\gamma+\rho), & \text { if } \rho<0, \gamma+\rho \neq 0 .\end{cases}
$$

Here, $\lim _{t \rightarrow \infty} A(t)=0$ and $|A(t)| \in R V_{\rho}$ with $\rho \leq 0$, see Lemma 5.2 of Draisma et al. (1999) and Corollary 2.3.5 in de Haan and Ferreira (2006). By appropriate choices of $a(t)$ and $A(t)$ in Eq. 2.2, for any $\varepsilon>0, \delta>0$ there exists $t_{0}=t_{0}(\varepsilon, \delta)$ such that

$$
\left|\frac{\frac{U(t x)-U(t)}{a(t)}-D_{\gamma}(x)}{A(t)}-k_{\gamma, \rho}(x)\right| \leq \varepsilon x^{\gamma+\rho} \max \left(x^{\delta}, x^{-\delta}\right)
$$

for all $\min (t, t x) \geq t_{0}$. For more details, see Theorem 2.3.6 of de Haan and Ferreira (2006). We may use the following alternative representation of Eq. 2.2:

$$
\frac{U(t x)-U(t)}{a(t)}=D_{\gamma}(x)+A(t) k_{\gamma, \rho}(x)+o(A(t))
$$

as $t \rightarrow \infty$.

For technical convenience, let $Y_{1}, \ldots, Y_{n}$ be independent random variables with the standard Pareto d.f $F_{Y}(y)=1-1 / y, y \geq 1$. Let $Y_{1, n} \leq Y_{2, n} \leq \cdots \leq Y_{n, n}$ denote the associated increasing order statistics. Then

$$
\left\{Y_{n-i, n} / Y_{n-k, n}\right\}_{i=0}^{k-1} \stackrel{d}{=}\left\{Y_{k-i, k}\right\}_{i=0}^{k-1}, \quad\left\{X_{n-i, n}\right\}_{i=0}^{n-1} \stackrel{d}{=}\left\{U\left(Y_{n-i, n}\right)\right\}_{i=0}^{n-1}
$$

cf. de Haan (1994). Note that for $m=m(n) \rightarrow \infty, m / n \rightarrow 0$ as $n \rightarrow \infty$, we have

$$
Y_{n-m, n} \stackrel{P}{\sim} n / m, \quad Y_{n-2 m, n} / Y_{n-m, n} \stackrel{P}{\sim} 1 / 2
$$

as $n \rightarrow \infty$. Moreover,

$$
Y_{n-2 m, n} / Y_{n-m, n} \stackrel{d}{=}:(1 / 2) \exp \left\{Q_{n} / \sqrt{2 m}\right\}, Y_{n, n} / Y_{n-m, n} \stackrel{d}{=} \max _{1 \leq i \leq m} Y_{i}=: m S_{n}
$$

where $Q_{n}$ converges in distribution to a standard normal random variable $Q$ and $S_{n}$ converges in distribution to $S$, a unit Fréchet random variable, i.e. a random variable with the d.f $\exp (-1 / y), y>0$. Obviously, $Q$ is independent of $S$. 
Theorem 2.3 derives asymptotic representations for $\widehat{\gamma}_{n}^{\text {Invwh }}\left(k_{0}, k\right)$.

Theorem 2.3 Suppose that Eq. 2.2 holds for $\gamma \in \mathbb{R}$ and integers $k_{0}$, $k$ and $m$ satisfy Eq. 1.16, and

$$
A\left(n / k_{0}\right) \log \left(k_{0} / k\right) \rightarrow 0, A\left(n / k_{0}\right) \log k_{0} \rightarrow 0
$$

as $n \rightarrow \infty$. Then $\widehat{\gamma}_{n}^{\text {Invwh }}\left(k_{0}, k\right)$ has the following asymptotic representations:

(1) For $\gamma>0$,

$$
\begin{aligned}
\widehat{\gamma}_{n}^{\text {Invwh }}\left(k_{0}, k\right)= & \gamma+\frac{\gamma P_{n}}{\sqrt{k_{0}}}+\frac{\left(2^{\gamma}-1\right)\left(-S_{n}^{-\gamma}\right)}{k_{0}^{\gamma} \log 2} \\
& +\left[\frac{\gamma \rho}{(1-\rho)(\gamma+\rho)} I_{\{\gamma \neq-\rho\}}+\frac{\gamma}{(1+\gamma)^{2}} \cdot I_{\{\gamma=-\rho\}}\right] \cdot I_{\{\rho<0\}} \\
& \cdot A\left(\frac{n}{k_{0}}\right)+I_{\{\rho=0\}} \cdot A\left(\frac{n}{k_{0}}\right)+\frac{\gamma}{1+\gamma} Y_{k-k_{0}, k}^{-\gamma}+o_{P}\left(\frac{1}{\sqrt{k}_{0}}\right) \\
& +o_{P}\left(k_{0}^{-\gamma}\right)+o_{P}\left(A\left(\frac{n}{k_{0}}\right)\right)+o_{P}\left(\left(\frac{k_{0}}{k}\right)^{\gamma}\right),
\end{aligned}
$$

where

$$
P_{n}:=\sqrt{k_{0}} \cdot\left[\frac{1}{k_{0}} \sum_{i=0}^{k_{0}-1} \log \left(\frac{Y_{n-i, n}}{Y_{n-k_{0}, n}}\right)-1\right] \stackrel{d}{\rightarrow} P \sim N(0,1)
$$

and $S_{n}$ and $P_{n}$ are asymptotically independent.

(2) For $\gamma=0$,

$$
\begin{aligned}
\widehat{\gamma}_{n}^{\text {Invwh }}\left(k_{0}, k\right)= & \frac{1}{\log Y_{k-k_{0}, k}}-\frac{1}{\log \left(k_{0}+1\right) / 2}+\frac{\log S_{n}}{\log ^{2}\left(k_{0}+1\right) / 2} \\
& +\left\{\frac{1}{\log Y_{k-k_{0}, k}}+\frac{1}{\log \left(k_{0}+1\right) / 2}\right\} \\
& \times\left\{O_{P}\left(A\left(\frac{n}{\left(k_{0}+1\right) / 2}\right)\right)+O_{P}\left(\frac{1}{\sqrt{\left(k_{0}+1\right) / 2}}\right)\right\} .
\end{aligned}
$$

(3) For $\gamma<0$,

$$
\begin{aligned}
\widehat{\gamma}^{\text {Invwh }}\left(k_{0}, k\right)= & \gamma+\frac{\left(2^{-\gamma}-1\right)\left(-S_{n}^{\gamma}\right)}{\log 2 \cdot k_{0}^{-\gamma}}-\frac{\gamma Q_{n}}{\sqrt{k_{0}} \log 2} \\
& +\left\{\frac{\gamma\left(2^{\rho}-1\right)}{(\gamma+\rho) \log 2} \cdot I_{\{\rho<0\}}+2^{\rho} \cdot I_{\{\rho=0\}}\right\} A\left(\frac{n}{k_{0}}\right)^{-\gamma} \\
& +\frac{\gamma}{1-\gamma} Y_{k-k_{0}, k}^{\gamma}+o_{P}\left(k_{0}^{\min (-1 / 2, \gamma)}\right)+o_{P}\left(\left(\frac{k_{0}}{k}\right)^{-\gamma}\right) .
\end{aligned}
$$


Theorem 2.4 Under the conditions given in Theorem 2.3, let

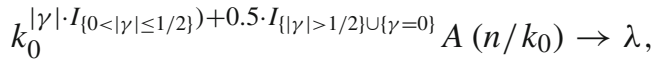

$$
\begin{aligned}
& k_{0}^{\left.|\gamma| \cdot I_{\{0<|\gamma| \leq 1 / 2\}}\right)+0.5 \cdot I_{\{|\gamma|>1 / 2\} \cup\{\gamma=0\}} / \log k_{0} / k \rightarrow 0,}
\end{aligned}
$$

where $\lambda \in \mathbb{R}$. We have

a. For $\gamma>0$,

(1) If $\gamma>1 / 2$ then $\sqrt{k}_{0}\left[\widehat{\gamma}_{n}^{\text {Invwh }}\left(k_{0}, k\right)-\gamma\right]$ converges in distribution to

$$
\begin{aligned}
\gamma P+\lambda \cdot\{ & {\left[\frac{\gamma \rho}{(1-\rho)(\gamma+\rho)} \cdot I_{\{\gamma \neq-\rho\}}+\frac{\gamma}{(1+\gamma)^{2}} \cdot I_{\{\gamma=-\rho\}}\right] } \\
& \left.\cdot I_{\{\rho<0\}}+I_{\{\rho=0\}}\right\},
\end{aligned}
$$

where $P \sim N(0,1)$.

(2) If $\gamma=1 / 2$ then $\sqrt{k}_{0}\left[\widehat{\gamma}_{n}^{\text {Invwh }}\left(k_{0}, k\right)-\gamma\right]$ converges in distribution to a sum of a normal and Weibull random variables, namely to

$$
\begin{aligned}
\gamma P+\frac{\left(2^{\gamma}-1\right)}{\log 2} Z+\lambda \cdot\{ & {\left[\frac{\gamma \rho}{(1-\rho)(\gamma+\rho)} \cdot I_{\{\gamma \neq-\rho\}}+\frac{\gamma}{(1+\gamma)^{2}} \cdot I_{\{\gamma=-\rho\}}\right] } \\
& \left.\cdot I_{\{\rho<0\}}+I_{\{\rho=0\}}\right\},
\end{aligned}
$$

where $P$ is a standard normal variable independent of $Z$, a Weibull random variable with shape parameter $1 / \gamma$.

(3) If $0<\gamma<1 / 2$ then $k_{0}^{\gamma}\left[\widehat{\gamma}_{n}^{\text {Invwh }}\left(k_{0}, k\right)-\gamma\right]$ converges in distribution to

$$
\begin{aligned}
\frac{2^{\gamma}-1}{\log 2} Z+\lambda \cdot\{ & {\left[\frac{\gamma \rho}{(1-\rho)(\gamma+\rho)} \cdot I_{\{\gamma \neq-\rho\}}+\frac{\gamma}{(1+\gamma)^{2}} \cdot I_{\{\gamma=-\rho\}}\right] } \\
& \left.\cdot I_{\{\rho<0\}}+I_{\{\rho=0\}}\right\},
\end{aligned}
$$

where $Z$ is a Weibull random variable with shape parameter $1 / \gamma$.

b. For $\gamma=0$, we have $\left(\log k_{0}\right)^{2}\left[\widehat{\gamma}_{n}^{\text {Invwh }}\left(k_{0}, k\right)+\frac{1}{\log \left(k_{0} / 2\right)}\right]$ converging in distribution to $Z$, a Gumbel random variable.

c. For $\gamma<0$,

(1) If $\gamma<-1 / 2$ then $\sqrt{k}_{0}\left[\widehat{\gamma}_{n}^{\text {Invwh }}\left(k_{0}, k\right)-\gamma\right]$ converges in distribution to

$$
\frac{\gamma}{\log 2}(-Q)+\lambda \cdot\left\{\frac{\gamma\left(2^{\rho}-1\right)}{(\gamma+\rho) \log 2} \cdot I_{\{\rho<0\}}+2^{\rho} \cdot I_{\{\rho=0\}}\right\},
$$

where $Q \sim N(0,1)$. 
(2) If $\gamma=-1 / 2$ then $\sqrt{k}_{0}\left[\widehat{\gamma}_{n}^{\text {Invwh }}\left(k_{0}, k\right)-\gamma\right]$ converges in distribution to a convolution of a normal and Weibull random variables, namely to

$$
\frac{2^{-\gamma}-1}{\log 2} Z-\frac{\gamma Q}{\log 2}+\lambda \cdot\left\{\frac{\gamma\left(2^{\rho}-1\right)}{(\gamma+\rho) \log 2} \cdot I_{\{\rho<0\}}+2^{\rho} \cdot I_{\{\rho=0\}}\right\},
$$

where $Q$ is a standard normal variable independent of $Z$, a Weibull random variable with shape parameter $-1 / \gamma$.

(3) If $-1 / 2<\gamma<0$ then $k_{0}^{-\gamma}\left[\widehat{\gamma}_{n}^{\text {Invwh }}\left(k_{0}, k\right)-\gamma\right]$ converges in distribution to

$$
\frac{2^{-\gamma}-1}{\log 2} Z+\lambda \cdot\left\{\frac{\gamma\left(2^{\rho}-1\right)}{(\gamma+\rho) \log 2} \cdot I_{\{\rho<0\}}+2^{\rho} \cdot I_{\{\rho=0\}}\right\},
$$

where $Z$ is a Weibull random variable with shape parameter $-1 / \gamma$.

Theorem 2.4 shows that the asymptotic distribution of $k_{0}^{\min (-\gamma, 1 / 2)}\left[\widehat{\gamma}_{n}^{\operatorname{Invwh}}\left(k_{0}, k\right)-\gamma\right]$ depends on the parameters $\gamma, \rho$ and $\lambda$. A bias corrected estimator may be established if the parameters $\rho$ and $\lambda$ are known. To avoid depending on the unknown parameters, we consider a particular case of the second order auxiliary function $A(t) \sim c t^{\rho}$ with $\rho<0$ and $c \neq 0$. This may help us to find the optimal choice for $k_{0}$ and to establish a bias corrected estimator.

Before discussing the optimal choice of $k_{0} \equiv k_{0}^{(o p t)}$ that minimizes the asymptotic mean squared error of $\widehat{\gamma}_{n}^{\text {Invwh }}\left(k_{0}, k\right)$ for the case of $A(t) \sim c t^{\rho}$, we need to introduce some notations:

$$
\begin{gathered}
\sigma^{2}(\gamma)= \begin{cases}\frac{\gamma^{2}}{\log ^{2} 2}, & \gamma<-1 / 2, \\
\left(\frac{2^{|\gamma|-1}}{\log 2}\right)^{2}\left[\Gamma(1+2|\gamma|)-\Gamma^{2}(1+|\gamma|)\right], & 0<|\gamma|<1 / 2, \\
\gamma^{2}, & \gamma>1 / 2,\end{cases} \\
c_{1}=\frac{\gamma}{1+|\gamma|}, \quad c_{2}= \begin{cases}\frac{\gamma \rho}{(1-\rho)(\gamma+\rho)} \cdot I_{\{\gamma \neq-\rho\}}+\frac{\gamma}{(1+\gamma)^{2}} \cdot I_{\{\gamma=-\rho\}}, & \gamma>0, \\
\frac{\gamma}{(\gamma+\rho) \log 2}, & \gamma<0,\end{cases}
\end{gathered}
$$

and

$$
c_{3}=\frac{-\sigma(\gamma) \cdot \Gamma(1+|\gamma|)}{\sqrt{\Gamma(1+2|\gamma|)-\Gamma^{2}(1+|\gamma|)}} .
$$


Theorem 2.5 Suppose that Eq. 2.2 holds with $A(t) \sim c t^{\rho}, c \neq 0$ and integers $k_{0}$, $k$ and $m$ satisfy Eqs. 1.16 and 2.5. For $|\gamma| \notin\{0,1 / 2\}$, the optimal sample fraction $k_{0}^{(o p t)}$ is given by:

(1) For $|\gamma|>1 / 2$, let

$$
\begin{aligned}
& k_{0}^{(1)}=\left\{\frac{\sigma^{2}(\gamma)}{2|\gamma| c_{1}^{2}}\right\}^{\frac{1}{2|\gamma|+1}} k^{\frac{2|\gamma|}{2|\gamma|+1}}, k_{0}^{(2)}=\left\{\frac{\sigma^{2}(\gamma)}{2|\rho| c^{2} c_{2}^{2}}\right\}^{\frac{1}{2|\rho|+1}} n^{\frac{2|\rho|}{2|\rho|+1}}, \\
& k_{0}^{(3)}=\left(\frac{c^{2} c_{2}^{2}}{c_{1}^{2}}\right)^{\frac{1}{2(|\gamma|+\rho)}} k^{\frac{|\gamma|}{|\gamma|+\rho}} \cdot n^{\frac{\rho}{\gamma \mid+\rho}} .
\end{aligned}
$$

Then the sequence $k_{0}^{(o p t)}$ that minimizes $\operatorname{MSE} E_{\infty}\left(\widehat{\gamma}_{n}^{\text {Invwh }}\left(k_{0}, k\right)\right)$ is:

(i) $\operatorname{For}|\gamma| \leq|\rho|, k_{0}^{(o p t)}=\left[k_{0}^{(1)}\right]$;

(ii) $\operatorname{For}|\gamma|>|\rho|$,

(a) If $k \ll n^{\frac{2|\rho|}{2|\rho|+1} \cdot \frac{2|\gamma|+1}{2|\gamma|}}$ then $k_{0}^{(o p t)}=\left[k_{0}^{(1)}\right]$;

(b) If $k \gg n^{\frac{2|\rho|}{2|\rho|+1} \cdot \frac{2|\gamma|+1}{2|\gamma|}}$ then $k_{0}^{(o p t)}=\left[k_{0}^{(2)}\right]$ for $c \gamma>0$ and $k_{0}^{(o p t)}=$ $\left[k_{0}^{(3)}\right]$ for $c \gamma<0$;

(c) If $k \sim D n^{\frac{2|\rho|}{2|\rho|+1} \cdot \frac{2|\gamma|+1}{2|\gamma|}}$ with $D \neq 0$ then $k_{0}^{(\text {opt })} \sim D_{1} n^{\frac{2|\rho|}{2|\rho|+1}}$ with $D_{1} \equiv$ $D_{1}(\gamma, \rho, c, D)$ satisfying

$$
\begin{aligned}
\frac{2|\gamma| c_{1}^{2}}{D^{2|\gamma|}} & \cdot D_{1}^{2|\gamma|+1}+\frac{2 c_{1} c_{2} c(|\gamma|+|\rho|)}{D^{|\gamma|}} \\
& \cdot D_{1}^{|\gamma|+|\rho|+1}+2|\rho|\left(c_{2} c\right)^{2} \cdot D_{1}^{2|\rho|+1}=\sigma^{2}(\gamma) .
\end{aligned}
$$

(2) For $0<|\gamma|<1 / 2$, let

$$
k_{0}^{(11)}=\left\{\frac{\sigma^{2}(\gamma)+c_{3}^{2}}{c_{1}^{2}}\right\}^{\frac{1}{4|\gamma|}} k^{1 / 2}, k_{0}^{(21)}=\left\{\frac{|\gamma|\left(\sigma^{2}(\gamma)+c_{3}^{2}\right)}{|\rho|\left(c c_{2}\right)^{2}}\right\}^{\frac{1}{2(|\rho|+|\gamma|)}} n^{\frac{|\rho|}{|\gamma|+|\rho|}}
$$

and

$$
k_{0}^{(31)}=\left(\frac{c_{3}^{2}}{c^{2} c_{2}^{2}}\right)^{1 / 2(|\gamma|+|\rho|)} \cdot n^{|\rho| /(|\gamma|+|\rho|)} .
$$

Then the sequence $k_{0}^{(o p t)}$ that minimizes $\operatorname{MS} E_{\infty}\left(\widehat{\gamma}_{n}^{\text {Invwh }}\left(k_{0}, k\right)\right)$ is:

(i) $F o r|\gamma| \leq|\rho|, k_{0}^{(o p t)}=\left[k_{0}^{(11)}\right]$; 
(ii) $\operatorname{For}|\gamma|>|\rho|$,

(a) If $k \ll n^{\frac{2|\rho|}{|\gamma|+|\rho|}}$ then $k_{0}^{(o p t)}=\left[k_{0}^{(11)}\right]$;

(b) If $k \gg n^{\frac{2|\rho|}{|\gamma|+|\rho|}}$ then $k_{0}^{(o p t)}=\left[k_{0}^{(31)}\right]$ for $c \gamma>0$ and $k_{0}^{(o p t)}=\left[k_{0}^{(3)}\right]$ for $c \gamma<0$

(c) If $k \sim D n^{\frac{2|\rho|}{|\gamma|+|\rho|}}$ with $D \neq 0$ then $k_{0}^{(o p t)} \sim D_{1} n^{\frac{2|\rho|}{|\gamma|+|\rho|}}$ with $D_{1} \equiv$ $D_{1}(\gamma, \rho, c, D)$ satisfying

$$
\begin{aligned}
\frac{c_{1}^{2}}{D^{2|\gamma|}} & \cdot D_{1}^{4|\gamma|}+\frac{c_{1} c_{2} c(1+|\rho / \gamma|)}{D^{|\gamma|}} \cdot D_{1}^{3|\gamma|+|\rho|}+|\rho / \gamma|\left(c_{2} c\right)^{2} \\
& \cdot D_{1}^{2|\gamma|+2|\rho|}-(1-|\rho / \gamma|) \cdot c c_{2} c_{3} \cdot D_{1}^{|\gamma|+|\rho|}=\sigma^{2}(\gamma)+c_{3}^{2} .
\end{aligned}
$$

By the arguments of Theorem 2.5, we deduce the following result.

Corollary 2.1 Suppose that Eq. 2.2 holds for $|\gamma|>1 / 2$ with $A(t) \sim c^{\rho}, c \neq 0$, $\rho<0$ and integers $k_{0}, k$ and $m$ satisfy Eqs. 1.16 and 2.5. Then when $|\gamma| \leq|\rho|$, or $|\gamma|>|\rho|$ and $k \ll n^{\frac{|\rho|(2|\gamma|+1)}{|\gamma|(2|\rho|+1)}}$, the optimal fraction $k_{0}^{(o p t)}=\left[k_{0}^{(1)}\right]$. Moreover, the asymptotical bias error of $\widehat{\gamma}_{n}^{\text {Invwh }}\left(k_{0}, k\right)$ is $\sigma(\gamma) / \sqrt{2|\gamma| k_{0}^{(1)}}$.

By Corollary 2.1, we obtain a bias corrected estimator for $|\gamma|>1 / 2$.

\section{Corollary 2.2 Set}

$$
\widetilde{\gamma}_{n}^{\text {Invwh }}\left(k_{0}^{(1)}, k\right)=\widehat{\gamma}_{n}^{\text {Invwh }}\left(k_{0}^{(1)}, k\right)-\frac{\sigma\left(\widehat{\gamma}_{n}^{\text {Invwh }}\left(k_{0}^{(1)}, k\right)\right)}{\sqrt{2\left|\widehat{\gamma}_{n}^{\text {Invwh }}\left(k_{0}^{(1)}, k\right)\right| k_{0}^{(1)}}} .
$$

Under the conditions of Corollary 2.1, $\sqrt{k_{0}^{(1)}}\left\{\widetilde{\gamma}_{n}^{\text {Invwh }}\left(k_{0}^{(1)}, k\right)-\gamma\right\}$ converges to a normal random variable with mean zero and variance $\Sigma_{0}$, where

$$
\Sigma_{0}= \begin{cases}\gamma^{2}, & \gamma>1 / 2, \\ \gamma^{2} / \log ^{2} 2, & \gamma<-1 / 2 .\end{cases}
$$

Similarly, we have the following two results for $0<|\gamma|<1 / 2$.

Corollary 2.3 Suppose that Eq. 2.2 holds for $|\gamma|<1 / 2$ with $A(t) \sim c t^{\rho}, c \neq 0$, $\rho<0$ and integers $k_{0}, k$ and $m$ satisfy Eqs. 1.16 and 2.5. Then when $|\gamma| \leq|\rho|$, or $|\gamma|>|\rho|$ and $k \ll n^{\frac{2|\rho|}{|\gamma|+|\rho|}}$, the optimal fraction $k_{0}^{(o p t)}=\left[k_{0}^{(11)}\right]$. Moreover, the asymptotical bias error of $\widehat{\gamma}_{n}^{\text {Invwh }}\left(k_{0}, k\right)$ is $\left\{c_{3}+\sqrt{c_{3}^{2}+\sigma^{2}(\gamma)}\right\} /\left(k_{0}^{(11)}\right)^{|\gamma|}$. 
Corollary 2.4 Set

$$
\begin{aligned}
\widetilde{\gamma}_{n}^{\text {Invwh }}\left(k_{0}^{(11)}, k\right)= & \widehat{\gamma}_{n}^{\text {Invwh }}\left(k_{0}^{(11)}, k\right) \\
& -\left\{\widehat{c_{3}}+\sqrt{\widehat{c}_{3}^{2}+\sigma^{2}\left(\widehat{\gamma}_{n}^{\text {Invwh }}\left(k_{0}^{(11)}, k\right)\right)}\right\} \\
& /\left(k_{0}^{(11)}\right)^{\left|\widehat{\gamma}_{n}^{\text {Invwh }}\left(k_{0}^{(11)}, k\right)\right|} .
\end{aligned}
$$

Under the conditions of Corollary 2.3, $\left(k_{0}^{(11)}\right)^{\left|\widehat{\gamma}_{n}^{\text {Inwh }}\left(k_{0}^{(11)}, k\right)\right|}\left(\widetilde{\gamma}_{n}^{\text {Invwh }}\left(k_{0}^{(11)}, k\right)-\gamma\right\}$ converges in distribution to a random variable with mean zero and variance $\sigma^{2}(\gamma)$, where $\sigma^{2}(\gamma)$ is the one defined in Eq. 2.9, and $\widehat{c_{3}}$ is the one defined in Eq. 2.10 with $\gamma$ replaced by $\widehat{\gamma}_{n}^{\text {Invwh }}\left(k_{0}^{(11)}, k\right)$.

\section{Simulation studies}

In this section, we present some simulation results, mainly comparing the finite sample behavior of the proposed estimator with other estimators discussed in Section 1.

Firstly, we compare the behavior of the Weiss-Hill estimator in Eq. 1.9 with the location invariant Hill estimator in Eq. 1.13 and the location invariant Weiss-Hill estimator in Eq. 1.15. Obviously, the new location invariant Weiss-Hill estimator performs very well in finite sample simulations. The random samples are from the three parameter Burr distribution:

$$
\operatorname{Burr}(x)=1-\left[1+(x-\mu)^{-\rho / \gamma}\right]^{1 / \rho}, x>\mu, \mu \in \mathbb{R}, \gamma>0, \rho<0
$$

with $\gamma=1, \rho=-0.8$, corresponding to $\gamma+\rho>0$. As expected the sample paths of the location invariant Weiss-Hill estimator are more stable than those of the WeissHill estimator. Moreover, we find that the location invariant Hill estimator and the location invariant Weiss-Hill estimator are comparable, and behave better than the Weiss-Hill estimator. For more interesting results, see Fig. 1.

Secondly, we compare the asymptotic mean squared errors of the location invariant Weiss-Hill estimator in Eq. 1.15 and the location invariant moment estimator in Eq. 1.14. Figure 2 shows that the mean squared errors of the location invariant WeissHill estimator are less than those of the location invariant moment estimator for at least $\gamma \leq-1$. This may be due to the fact that we choose $k=[0.1 n], k_{0}=[0.1 k]$, where $n$ is the sample size. Here, random samples are taken from the generalized Pareto distribution:

$$
G P D_{\gamma, \mu}(y)= \begin{cases}1-\{1+\gamma(y-\mu)\}^{-1 / \gamma}, & \text { for } 1+\gamma(y-\mu)>0, \text { if } \gamma \neq 0, \mu \in \mathbb{R}, \\ 1-\exp \{-(y-\mu)\}, & \text { for } y>\mu, \text { if } \gamma=0, \mu \in \mathbb{R} .\end{cases}
$$




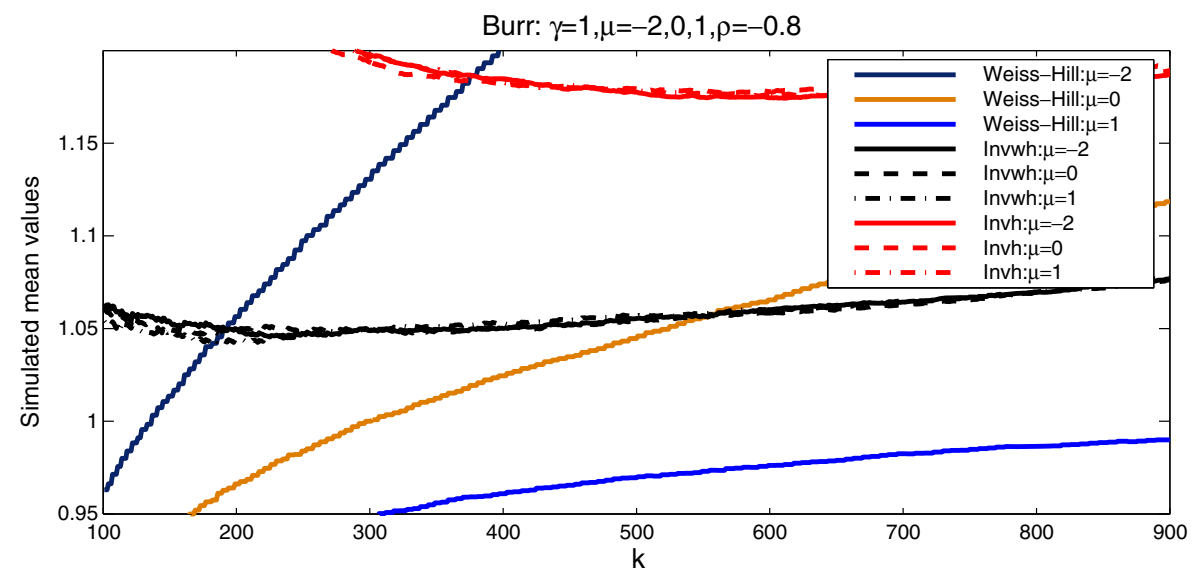

Fig. 1 Simulated mean values of the following estimators: Weiss-Hill estimator in Eq. 1.9, location invariant Hill estimator in Eq. 1.13 and location invariant Weiss-Hill estimator in Eq. 1.15. Random samples are from the Burr distribution. Sample size is 1000 with the number of replications 1000

Figure 3 compares finite sample behaviors of various tail index estimators. The considered estimators are: $\widehat{\gamma}_{n}^{\operatorname{mixM}}(k)$ in Eq. 1.3, $\widehat{\gamma}_{n}^{C H}(k)$ in Eq. 1.4, $\widehat{\gamma}_{n}^{C M}(k)$ in Eq. 1.5, $\widehat{\gamma}_{n}^{\text {Invm }}\left(k_{0}, k\right)$ in Eq. 1.14 and $\widehat{\gamma}_{n}^{\text {Invwh }}$ in Eq. 1.15. Random samples are from the Burr distribution with $\mu=0, \gamma=0.5$ and $\rho=-0.5$, corresponding to $\gamma+\rho=0$. Sample size is $n=1000$ with the number of replications equals to 1000 , and $k=l, l+1, \cdots, n-l-1$ with $l=100$. The simulations show that the finite sample behavior of the location invariant Weiss-Hill estimator proposed in

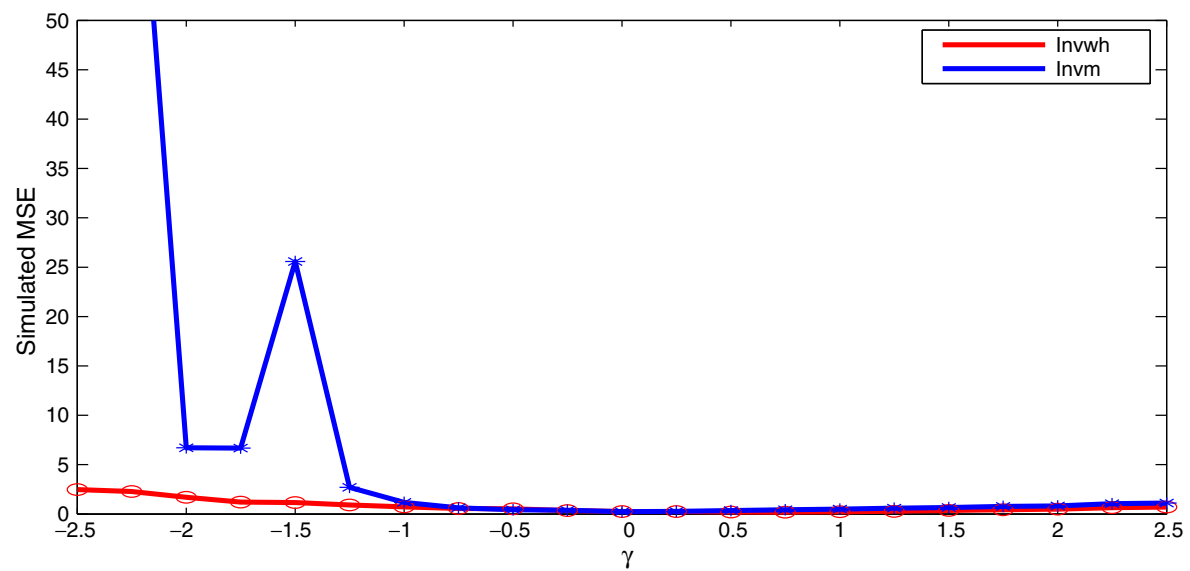

Fig. 2 Simulated mean squared errors of the following estimators: moment estimator in Eq. 1.14 and Weiss-Hill estimator in Eq. 1.15. Random samples are from the GPD. Sample size is $n=1000$ with $k=[0.1 n], k_{0}=[0.1 k]$. The number of replications is 1000 

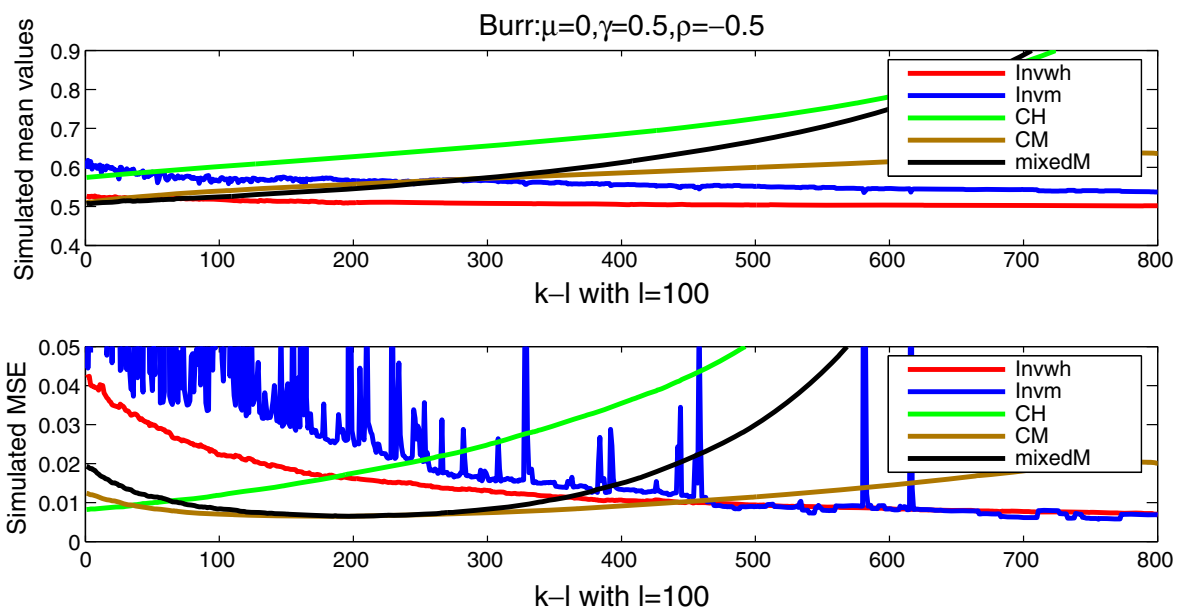

Fig. 3 Simulated mean values and mean squared errors of the following estimators: $\widehat{\gamma}_{n}^{\text {mix }} M_{(k)}$ in Eq. 1.3, $\widehat{\gamma}_{n}^{C H}(k)$ in Eq. 1.4, $\widehat{\gamma}_{n}^{C M}(k)$ in Eq. 1.5, $\widehat{\gamma}_{n}^{\text {Invm }}\left(k_{0}, k\right)$ in Eq. 1.14 and $\widehat{\gamma}_{n}^{\text {Invwh }}$ in Eq. 1.15. Random samples are from the Burr distribution with $\mu=0, \gamma=0.5$ and $\rho=-0.5$. Sample size is 1000 with the number of replications 1000

this paper is superior to that of other estimators. Furthermore, the location invariant Weiss-Hill estimator has smaller bias than the location invariant moment-type estimator $\widehat{\gamma}_{n}^{\text {Invm }}\left(k_{0}, k\right)$ in Eq. 1.14 and the corrected moment type estimator $\widehat{\gamma}_{n}^{C M}(k)$ in Eq. 1.5.
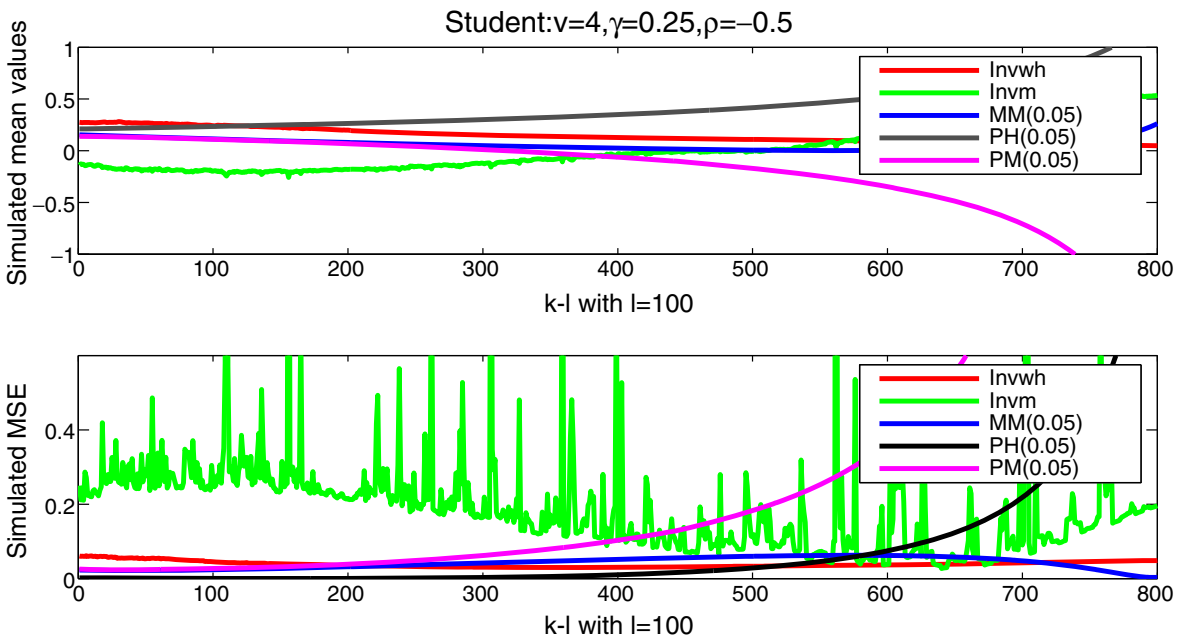

Fig. 4 Simulated mean values and mean squared errors of the following estimators: $\widehat{\gamma}_{n}^{P H}(0.05)$ in Eq. 1.10, $\widehat{\gamma}_{n}^{P M}(0.05)$ in Eq. 1.11, $\widehat{\gamma}_{n}^{M M}(0.05)$ in Eq. 1.12, $\widehat{\gamma}_{n}^{\text {Invm }}\left(k_{0}, k\right)$ in Eq. 1.14 and $\widehat{\gamma}_{n}^{\text {Invwh }}\left(k_{0}, k\right)$ in Eq. 1.15. Random samples are from the Student's $t$ distribution with degrees of freedom $v=4$. Sample size is 1000 with the number of replications 1000 


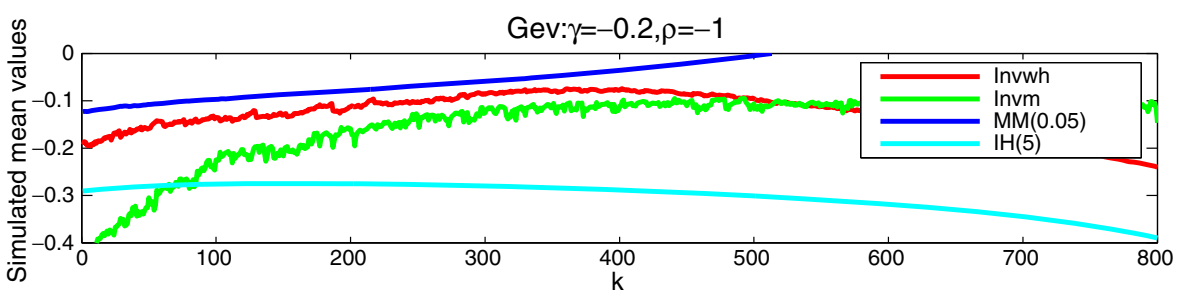

Gev: $\gamma=-0.2, \rho=-1$

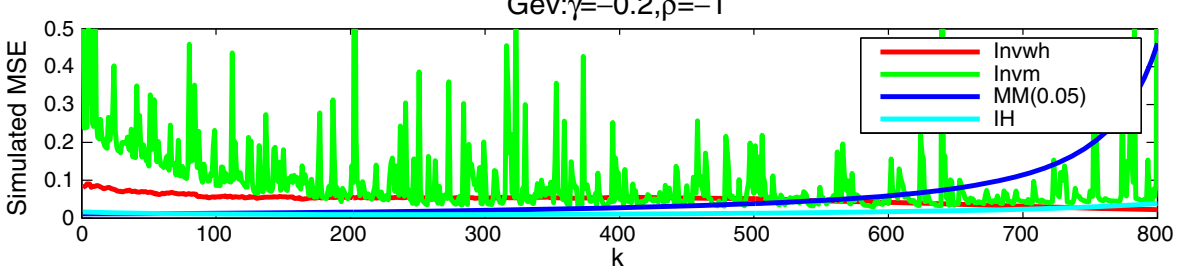

Fig. 5 Simulated mean values and mean squared errors of the following estimators: $\widehat{\gamma}_{n}^{I H}(5)$ in Eq. 1.6, $\widehat{\gamma}_{n}^{M M}(0.05)$ in Eq. 1.12, $\widehat{\gamma}_{n}^{I n v m}$ in Eq. 1.14 and $\widehat{\gamma}_{n}^{I n v w h}\left(k_{0}, k\right)$ in Eq. 1.15. Random samples are from the GEV distribution with $\gamma=-0.2, \rho=-1$. Sample size is 1000 with the number of replications 1000

Figures 4, 5, 6 and 7 compare the finite sample behaviors of simulated means and mean squared errors for several location invariant estimators discussed in Section 1. The considered estimators are: $\widehat{\gamma}_{n}^{P H}(p)$ in Eq. 1.10, $\widehat{\gamma}_{n}^{I H}(q)$ in Eq. 1.6, $\widehat{\gamma}_{n}^{P M}(p)$ in Eq. 1.11, $\widehat{\gamma}_{n}^{M M}(p)$ in Eq. 1.12, $\widehat{\gamma}_{n}^{\text {Invm }}\left(k_{0}, k\right)$ in Eq. 1.14 and $\widehat{\gamma}_{n}^{\text {Invwh }}\left(k_{0}, k\right)$ in
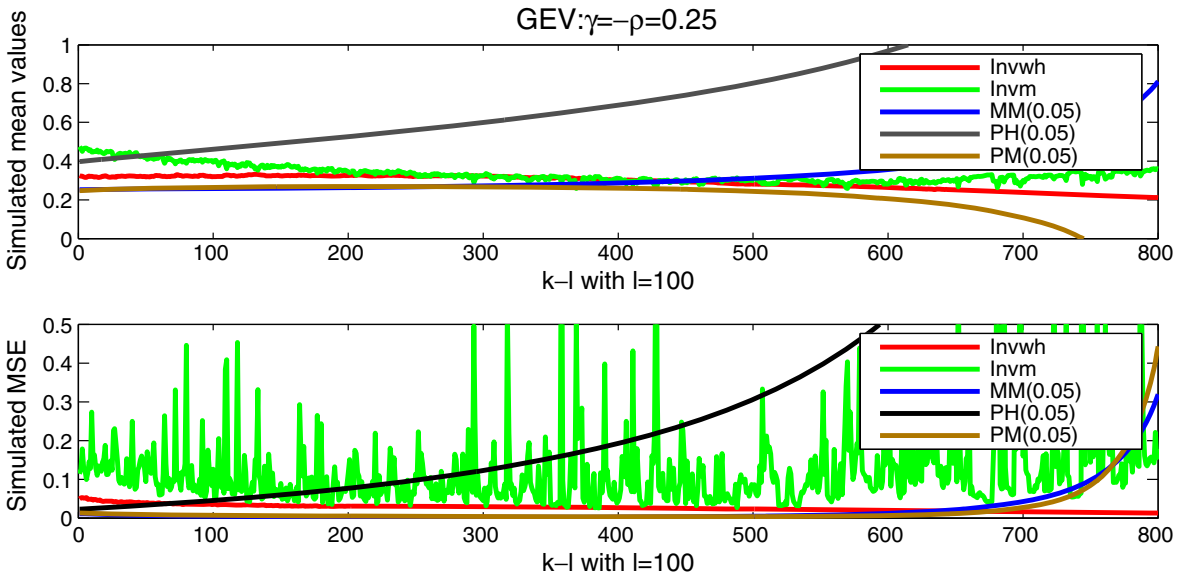

Fig. 6 Simulated mean values and mean squared errors of the following estimators: $\widehat{\gamma}_{n}^{P H}(0.05)$ in Eq. 1.10, $\widehat{\gamma}_{n}^{P M}(0.05)$ in Eq. 1.11, $\widehat{\gamma}_{n}^{M M}(0.05)$ in Eq. 1.12, $\widehat{\gamma}_{n}^{\text {Invm }}\left(k_{0}, k\right)$ in Eq. 1.14 and $\widehat{\gamma}_{n}^{\text {Invwh }}\left(k_{0}, k\right)$ in Eq. 1.15. Random samples are from the GEV distribution with $\gamma=0.25, \rho=-0.25$. Sample size is 1000 with the number of replications 1000 

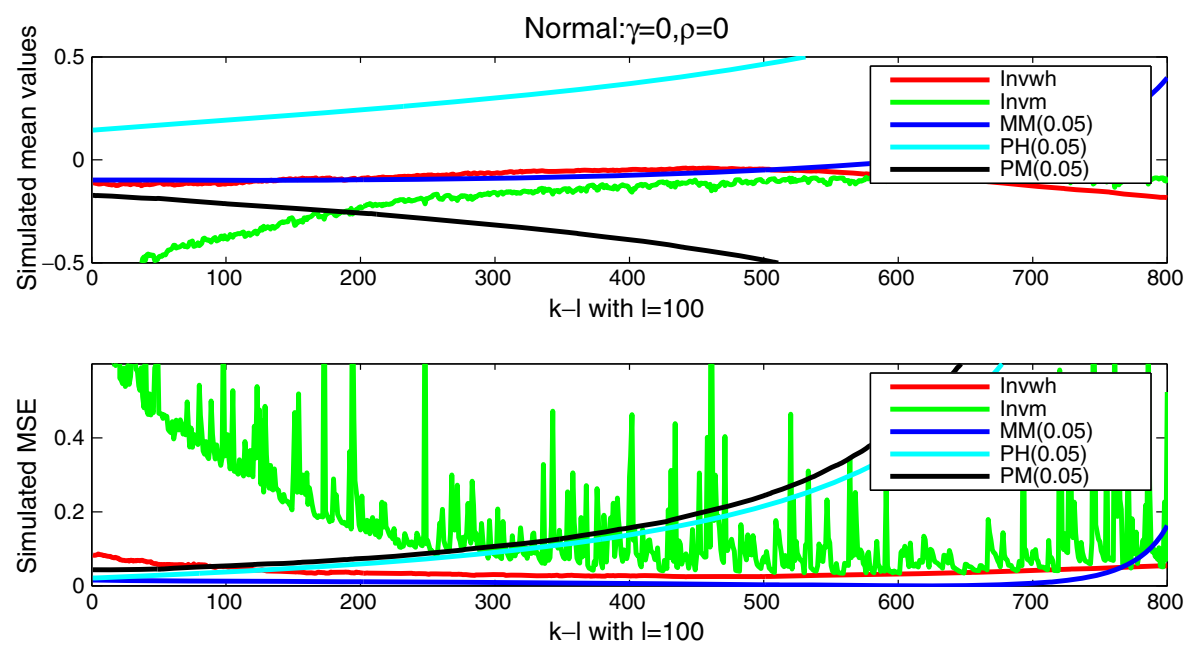

Fig. 7 Simulated mean values and mean squared errors of the following estimators: $\widehat{\gamma}_{n}^{P H}(0.05)$ in Eq. 1.10, $\widehat{\gamma}_{n}^{P M}(0.05)$ in Eq. 1.11, $\widehat{\gamma}_{n}^{M M}(0.05)$ in Eq. 1.12, $\widehat{\gamma}_{n}^{\text {Invm }}\left(k_{0}, k\right)$ in Eq. 1.14 and $\widehat{\gamma}_{n}^{\text {Invwh }}\left(k_{0}, k\right)$ in Eq. 1.15. Random samples are from the standard normal distribution, where $\gamma=0$ and $\rho=0$. Sample size is 1000 with the number of replications 1000

Eq. 1.15. The random samples are taken from the following distributions: Burr, Student's $t$, Normal, the GPD and the GEV. All sample sizes are $n=1000$ with the number of replications equals to $m=1000$. We take the initial $k_{0}$ as the optimal $k_{0}^{(o p t)}$ and then adjust the estimators according to Corollaries 2.2 and 2.4. We use an adjusted version of the algorithm proposed by Fraga Alves (2001a) to perform the simulations even though we know the true $\gamma$ and $\rho$ for the chosen distributions:

Step 1 Take the original $k_{0}^{*}$ as $k_{0}^{*}=\left[2 \cdot k^{2 / 3}\right], k=l, \ldots, n-l-1($ Here, $l=100)$;

Step 2 Compute the original estimator $\widehat{\gamma}_{n}^{\operatorname{Invwh}}\left(k_{0}^{*}, k\right)$;

Step 3 Compute the optimal sample fraction $\widehat{k}_{0}^{(o p t)}$ as:

$$
\widehat{k}_{0}^{(o p t)}= \begin{cases}{\left[\widehat{k}_{0}^{(1)}\right],} & \text { for }|\widehat{\gamma}|>1 / 2+0.05, \\ {\left[\widehat{k}_{0}^{(11)}\right],} & \text { for } 0.05<|\widehat{\gamma}| \leq 1 / 2+0.05, \\ {\left[2^{4 / 3} k^{2 / 3}\right],} & \text { for }|\widehat{\gamma}| \leq 0.05,\end{cases}
$$

where $\widehat{\gamma}$ is the one taken from Step 2, i.e. $\widehat{\gamma}_{n}^{\operatorname{Invwh}}\left(k_{0}^{*}, k\right)$;

Step 4 Repeat Step 2, compute $\widehat{\gamma}_{n}^{\text {Invwh }}\left(\widehat{k}_{0}^{(o p t)}, k\right)$; 
Step 5 Compute the bias corrected estimator $\left.\widetilde{\gamma}_{n}^{\text {Invwh }} \widehat{k}_{0}^{\left({ }^{(o p t)}\right.}, k\right)$ according to Corollaries 2.2 and 2.4 as

$$
\begin{aligned}
& \int \widehat{\gamma}_{n}^{\text {Invwh }}\left(\widehat{k}_{0}^{(o p t)}, k\right)-0.95 \frac{\sigma\left(\widehat{\gamma}_{n}^{W H}\left(\widehat{k}_{0}^{(o p t)}, k\right)\right)}{\sqrt{2\left|\widehat{\gamma}_{n}^{W H}\left(\widehat{k}_{0}^{(o p t)}, k\right)\right| \widehat{k}_{0}^{(o p t)}}}
\end{aligned}
$$

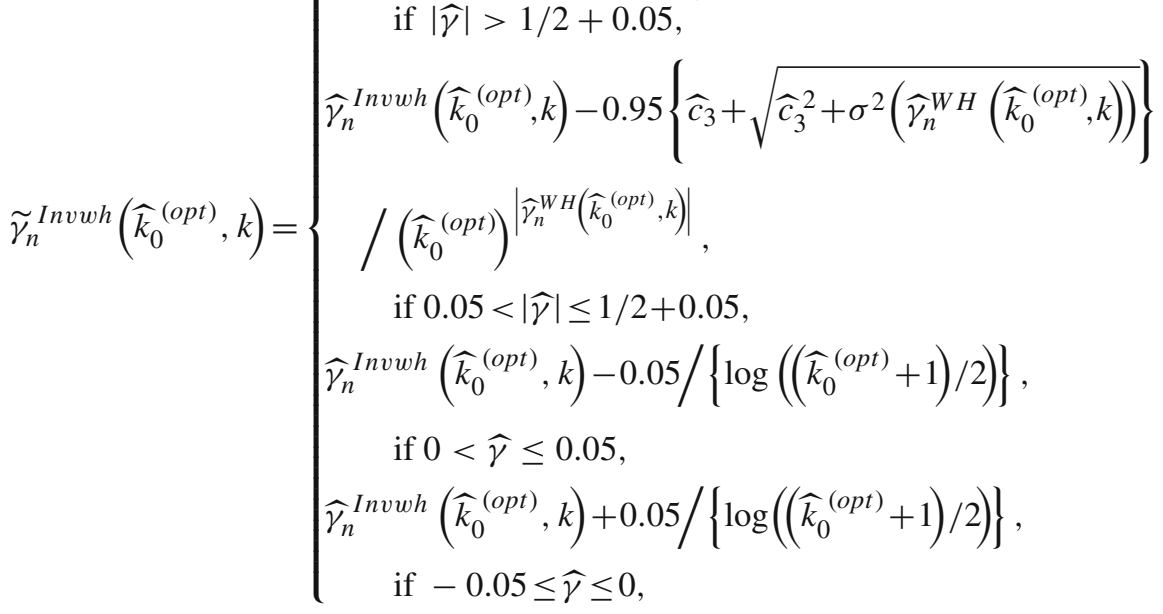

where $\widehat{\gamma}$ is the one taken from Step 4, i.e. $\left.\widehat{\gamma}_{n}^{\text {Invwh }} \widehat{k}_{0}^{(o p t)}, k\right)$.

Note that this algorithm has not used any information on the true values of $\gamma$ and $\rho$, so when selecting the optimal sample fraction and bias correction, we use the values 0.05 and 0.95 as a rule of thumb.

For $|\gamma|>1 / 2$, we find that simulated mean values are comparable for $\widehat{\gamma}^{\operatorname{Invm}}\left(k_{0}, k\right)$ and $\widehat{\gamma}^{\operatorname{Invwh}}\left(k_{0}, k\right)$, and that the corresponding biases are better than those of most of other estimators considered. So, we only consider the case of $|\gamma| \leq 1 / 2$. Figure 4 shows that the finite sample behaviors of the location invariant Weiss-Hill estimator, $\widehat{\gamma}_{n}^{\text {Invwh }}\left(k_{0}, k\right)$, and all the location invariant PORT estimators, including $\widehat{\gamma}_{n}^{P H}(0.05)$ in Eq. $1.10, \widehat{\gamma}_{n}^{P M}(0.05)$ in Eq. 1.11 and $\widehat{\gamma}_{n}^{M M}(0.05)$ in Eq. 1.12, are comparable. The finite sample behavior of the location invariant moment estimator, $\widehat{\gamma}_{n}^{\text {Invm }}\left(k_{0}, k\right)$, appears to be the worst. Here, the random sample is from the Student's $t$ distribution with degrees of freedom $v=4$. So, $\gamma=0.25$ and $\rho=-0.5$, corresponding to $\gamma+\rho<0$.

Finally, for $|\gamma|<1 / 2$, we study two cases: $\gamma$ smaller than zero and $\gamma+\rho=0$. Random samples are from the GEV distribution with $\gamma=-0.2, \rho=-1$ and from the GEV distribution with $\gamma=0.25, \rho=-0.25$. For the case of $\gamma=-0.2$, Fig. 5 shows that the simulated mean values are comparable for $\widehat{\gamma}^{\text {Invm }}\left(k_{0}, k\right)$ and $\widehat{\gamma}^{\text {Invwh }}\left(k_{0}, k\right)$. For the case of $\gamma+\rho=0$, there are two cases. For the GEV distribution with $\gamma=-\rho=0.25$, the paths of the simulated mean values are comparable for $\widehat{\gamma}^{\operatorname{Invm}}\left(k_{0}, k\right), \widehat{\gamma}^{\operatorname{Invwh}}\left(k_{0}, k\right)$ and $\widehat{\gamma}_{n}^{M M}(0.05)$, see Fig. 6. The asymptotic mean squared errors of $\widehat{\gamma}_{n}^{\text {Invwh }}\left(k_{0}, k\right), \widehat{\gamma}_{n}^{P M}(0.05)$ and $\widehat{\gamma}_{n}^{M M}(0.05)$ appear close to zero most of the time, see Fig. 6. For the normal distribution, $\gamma=\rho=0$. Simulations 
show that the two location invariant estimators: $\widehat{\gamma}_{n}^{\text {Invwh }}\left(k_{0}, k\right)$ and the location invariant mixed moment estimator, $\widehat{\gamma}_{n}^{M M}(0.05)$, behave very well. The sample paths for both estimators are stable and close to the true value. Furthermore, the corresponding asymptotic mean squared errors are close to zero most of the time, see Fig. 7.

\section{Proofs}

In this section, we prove the results provided in Section 2.

Proof of Theorem 2.1 Clearly,

$$
\begin{aligned}
& \widehat{\gamma}_{n}^{\text {Invwh }}\left(k_{0}, k\right)=\frac{1}{k_{0}} \sum_{i=0}^{k_{0}-1} \log \frac{X_{n-i, n}-X_{n-k, n}}{X_{n-k_{0}, n}-X_{n-k, n}}+\frac{1}{\log 2} \log \frac{X_{n, n}-X_{n-m+1, n}}{X_{n, n}-X_{n-2 m+1, n}} \\
& =\frac{1}{k_{0}} \sum_{i=0}^{k_{0}-1} \log \left(1+\frac{X_{n-i, n}-X_{n-k_{0}, n}}{X_{n-k_{0}, n}-X_{n-k, n}}\right) \\
& -\frac{1}{\log 2} \log \left(1+\frac{X_{n-m+1, n}-X_{n-2 m+1, n}}{X_{n, n}-X_{n-m+1, n}}\right) \\
& \stackrel{d}{=} \frac{1}{k_{0}} \sum_{i=0}^{k_{0}-1} \log \left\{1+\frac{U\left(Y_{n-i, n}\right)-U\left(Y_{n-k_{0}, n}\right)}{a\left(Y_{n-k_{0}, n}\right)}\right. \\
& \left.\frac{a\left(Y_{n-k_{0}, n}\right)}{U\left(Y_{n-k_{0}, n}\right)-U\left(Y_{n-k, n}\right)}\right\}-\frac{1}{\log 2} \log \\
& \left\{1+\frac{U\left(Y_{n-m+1, n}\right)-U\left(Y_{n-2 m+1, n}\right)}{a\left(Y_{n-m+1, n}\right)}\right. \\
& \left.\cdot \frac{a\left(Y_{n-m+1, n}\right)}{U\left(Y_{n, n}\right)-U\left(Y_{n-m+1, n}\right)}\right\} \\
& \triangleq \frac{1}{k_{0}} \sum_{i=0}^{k_{0}-1} \log \left(1+B_{1} \cdot B_{2}\right)-\frac{1}{\log 2} \log \left(1+B_{3} \cdot B_{4}\right),
\end{aligned}
$$

where

$$
\begin{aligned}
& B_{1}=\frac{U\left(Y_{n-i, n}\right)-U\left(Y_{n-k_{0}, n}\right)}{a\left(Y_{n-k_{0}, n}\right)}, \quad B_{2}=\frac{a\left(Y_{n-k_{0}, n}\right)}{U\left(Y_{n-k_{0}, n}\right)-U\left(Y_{n-k, n}\right)}, \\
& B_{3}=\frac{U\left(Y_{n-m+1, n}\right)-U\left(Y_{n-2 m+1, n}\right)}{a\left(Y_{n-m+1, n}\right)}, \quad B_{4}=\frac{a\left(Y_{n-m+1, n}\right)}{U\left(Y_{n, n}\right)-U\left(Y_{n-m+1, n}\right)} .
\end{aligned}
$$


We consider the following three cases.

(i) For $\gamma>0$.

Note that $a(t) \sim \gamma U(t)$ and $U(t) \in R V_{\gamma}$ for $\gamma>0$. By the Potter bound and Eq. 2.1, for arbitrary $x>0$ and $\varepsilon>0$, there exists $t_{0}>0$ such that as $\min (t, t x)>t_{0}$

$$
\begin{aligned}
& (1-\varepsilon) \frac{\min \left\{(1-\varepsilon) x^{\gamma-\varepsilon},(1+\varepsilon)^{-1} x^{\gamma+\varepsilon}\right\}-1}{\gamma}<\frac{U(t x)-U(t)}{a(t)} \\
& <(1+\varepsilon) \frac{\max \left\{(1+\varepsilon) x^{\gamma+\varepsilon},(1-\varepsilon)^{-1} x^{\gamma-\varepsilon}\right\}-1}{\gamma} .
\end{aligned}
$$

Moreover, $Y_{k-k_{0}, k}^{-\gamma} \stackrel{P}{\rightarrow} 0$ and $Y_{m, m} \stackrel{P}{\rightarrow} \infty$. So,

$$
\begin{aligned}
1+B_{1} \cdot B_{2} & =1+\frac{Y_{k_{0}-i, k_{0}}^{\gamma}-1}{\gamma} \cdot \frac{-\gamma}{Y_{k-k_{0}, k}^{-\gamma}-1} \cdot\left(1+o_{P}(1)\right) \\
& =Y_{k_{0}-i, k_{0}}^{\gamma}\left(1+o_{P}(1)\right)
\end{aligned}
$$

and

$$
1+B_{3} \cdot B_{4}=1-\frac{2^{-\gamma}-1}{Y_{m-1, m-1}^{\gamma}} \cdot\left(1+o_{P}(1)\right) \stackrel{P}{\rightarrow} 1 .
$$

So,

$$
\widehat{\gamma}_{n}^{\text {Invwh }}\left(k_{0}, k\right)=\frac{1}{k_{0}} \sum_{i=0}^{k_{0}-1} \log Y_{k_{0}-i, k_{0}}^{\gamma} \cdot\left(1+o_{P}(1)\right)+o_{P}(1) \rightarrow \gamma
$$

in probability.

(ii) For $\gamma=0$.

By Lemma 2.5(i) of Dekkers et al. (1989) and the condition 2.1, for arbitrary $\varepsilon>0$ there exists $t_{0}>0$ such that for $t>t_{0}$ and for all $x>1$,

$$
(1-\varepsilon) \frac{1-x^{-\varepsilon}}{\varepsilon}-\varepsilon<\frac{U(t x)-U(t)}{a(t)}<(1+\varepsilon) \frac{x^{\varepsilon}-1}{\varepsilon}+\varepsilon .
$$

So,

$$
1+B_{1} \cdot B_{2}=1-\frac{\log Y_{k_{0}-i, k_{0}}}{\log Y_{k-k_{0}, k}^{-1}}\left(1+o_{P}(1)\right)
$$

and

$$
1+B_{3} \cdot B_{4}=1+\frac{\gamma \log 2}{\log Y_{m-1, m-1}}\left(1+o_{P}(1)\right) \stackrel{P}{\rightarrow} 1
$$


So,

$$
{\widehat{\gamma_{n}}}_{n}^{\text {Invwh }}\left(k_{0}, k\right)=\frac{1}{k_{0}} \sum_{i=0}^{k_{0}-1} \frac{\log Y_{k_{0}-i, k_{0}}}{\log Y_{k-k_{0}, k}^{-1}}\left(1+o_{P}(1)\right)+o_{P}(1) \rightarrow 0
$$

in probability.

(iii) For $\gamma<0$.

The proof is similar to the case $\gamma>0$. Just note that $a(t) \sim-\gamma(U(\infty)-$ $U(t))$ and $U(\infty)-U(t) \in R V_{\gamma}$. Details are omitted here.

The proof is complete.

In order to prove Theorem 2.2, we need some auxiliary results. Lemma 4.1 is due to Dekkers et al. (1989).

Lemma 4.1 Suppose the integer sequence $k=k(n)$ satisfies $0<k=k(n) \leq n$ and $k /(\log n)^{\delta} \rightarrow \infty$ as $n \rightarrow \infty$ for some $\delta>0$.

1. If $F(x)=x^{\alpha}, 0<x<1$ for some $\alpha>0$ then

$$
\frac{1}{k} \sum_{i=1}^{k} \frac{X_{i, n}}{X_{k+1, n}} \rightarrow \frac{\alpha}{\alpha+1}
$$

almost surely.

2. If $F(x)=1-x^{-\alpha}, x>1$ for some $\alpha>2(1+\delta) / \delta$ then

$$
\frac{1}{k} \sum_{i=1}^{k} \frac{X_{n-i-1, n}}{X_{n-k, n}} \rightarrow \frac{\alpha}{\alpha-1}
$$

almost surely.

Proof See Lemma 2.3 of Dekkers et al. (1989).

For the order statistics from the standard Pareto distribution, we have

Lemma 4.2 Let $Y_{1, n} \leq Y_{2, n} \leq \cdots \leq Y_{n, n}$ be the order statistics from the standard Pareto distribution.

1. If $k / n \rightarrow 0$ and $k / \log n \rightarrow \infty$ then $(k / n) Y_{n-k+1, n} \rightarrow 1$ almost surely and $Y_{n-2 k+1, n} / Y_{n-k+1, n} \rightarrow 1 / 2$ almost surely.

2. If $k \rightarrow \infty$ and $k / n \rightarrow 0$ then $Y_{n, n} / Y_{n-k+1, n} \rightarrow \infty$ almost surely.

3. If $k_{0} / k \rightarrow 0, k / n \rightarrow 0, k_{0} / \log k \rightarrow \infty$ and $k / \log n \rightarrow \infty$ then $Y_{n-k+1, n} \rightarrow \infty$ almost surely and $Y_{n-k_{0}+1, n} / Y_{n-k+1, n} \rightarrow \infty$ almost surely.

Proof See Lemma 2 of He (2004). 
Lemma 4.3 Suppose Eq. 2.1 holds. As $t_{1} \rightarrow \infty$ and $t_{2} / t_{1} \rightarrow \infty$,

$$
\frac{\log \frac{U\left(t_{2} x\right)-U\left(t_{1}\right)}{U\left(t_{2}\right)-U\left(t_{1}\right)}}{\frac{a\left(t_{2}\right)}{U\left(t_{2}\right)-U\left(t_{1}\right)}} \rightarrow \begin{cases}\log x, & \gamma \geq 0 \\ \frac{x^{\gamma}-1}{\gamma}, & \gamma<0\end{cases}
$$

holds locally uniformly for arbitrary $x>0$. Furthermore, for arbitrary $\varepsilon>0$, there exists $N_{1}$ and $N_{2}$ such that $t_{1}>N_{1}$ and $t_{2} / t_{1}>N_{2}$, and for all $x \geq 1$,

(i) For $\gamma \geq 0$,

$$
(1-\varepsilon) \cdot \frac{1-x^{-\varepsilon}}{\varepsilon}-\varepsilon<\frac{\log \frac{U\left(t_{2} x\right)-U\left(t_{1}\right)}{U\left(t_{2}\right)-U\left(t_{1}\right)}}{\frac{a\left(t_{2}\right)}{U\left(t_{2}\right)-U\left(t_{1}\right)}}<(1+\varepsilon) \cdot \frac{x^{\varepsilon}-1}{\varepsilon}+\varepsilon
$$

(ii) For $\gamma<0$,

$$
\frac{(1+\varepsilon) \cdot x^{\gamma+\varepsilon}-1}{\gamma}<\frac{\log \frac{U\left(t_{2} x\right)-U\left(t_{1}\right)}{U\left(t_{2}\right)-U\left(t_{1}\right)}}{\frac{a\left(t_{2}\right)}{U\left(t_{2}\right)-U\left(t_{1}\right)}}<\frac{(1-\varepsilon) \cdot x^{\gamma-\varepsilon}-1}{\gamma} .
$$

Proof Note that

$$
\frac{U\left(t_{2} x\right)-U\left(t_{1}\right)}{U\left(t_{2}\right)-U\left(t_{1}\right)}=1+\frac{U\left(t_{2} x\right)-U\left(t_{2}\right)}{a\left(t_{2}\right)} \frac{a\left(t_{2}\right)}{U\left(t_{2}\right)-U\left(t_{1}\right)} .
$$

Now use Eq. 2.1 to derive the desired results as in Lemma 2.5 of Dekkers et al. (1989).

Proof of Theorem 2.2 We prove $\widehat{\gamma}_{n}^{H}\left(k_{0}, k\right) \stackrel{\text { a.s. }}{\longrightarrow} \gamma^{+}=\max (\gamma, 0)$ and $\widehat{\gamma}_{n}^{W}(m) \stackrel{\text { a.s. }}{\longrightarrow}$ $\gamma^{-}=\min (\gamma, 0)$. We consider the three cases: $\gamma>0, \gamma=0$ and $\gamma<0$.

(1) The case $\gamma>0$. We only need to show that both

$$
\begin{aligned}
& \frac{\widehat{\gamma}_{n}^{H}\left(k_{0}, k\right)}{a\left(Y_{n-k_{0}, n}\right) /\left[U\left(Y_{n-k_{0}, n}\right)-U\left(Y_{n-k, n}\right)\right]} \stackrel{a . s .}{\longrightarrow} 1, \\
& \frac{a\left(Y_{n-k_{0}, n}\right)}{U\left(Y_{n-k_{0}, n}\right)-U\left(Y_{n-k, n}\right)} \stackrel{a . s .}{\longrightarrow} \gamma
\end{aligned}
$$

and

$$
\widehat{\gamma}_{n}^{W}(m) \stackrel{\text { a.s. }}{\rightarrow} 0
$$


hold. By Lemmas 4.2 and 4.3, for given $\varepsilon>0$, one has

$$
\begin{gathered}
\frac{\widehat{\gamma}_{n}^{H}\left(k_{0}, k\right)}{a\left(Y_{n-k_{0}, n}\right) /\left[U\left(Y_{n-k_{0}, n}\right)-U\left(Y_{n-k, n}\right)\right]} \\
=\frac{1}{k_{0}} \sum_{i=0}^{k_{0}-1}\left\{\frac{\log \frac{U\left(Y_{n-i, n}\right)-U\left(Y_{n-k, n}\right)}{U\left(Y_{\left.n-k_{0}, n\right)-U\left(Y_{n-k, n}\right)}\right.}}{\left.\frac{a\left(Y_{n-k_{0}, n}\right)}{U\left(Y_{n-k_{0}, n}\right)-U\left(Y_{n-k, n}\right)}\right\}}\right. \\
\stackrel{a . s .}{<} \frac{1}{k_{0}} \sum_{i=0}^{k_{0}-1}\left\{\varepsilon+(1+\varepsilon) \cdot \frac{Y_{n-i, n}^{\varepsilon} / Y_{n-k_{0}, n}^{\varepsilon}-1}{\varepsilon}\right\}
\end{gathered}
$$

for all sufficiently large $n$. Since $Y_{i}^{\varepsilon}$ follows the d.f $1-1 / x^{1 / \varepsilon}, x>1$, we can apply Lemma 4.1 to find that, for $\varepsilon<\min \left\{\delta_{1} / 2\left(1+\delta_{1}\right), \delta_{2} / 2\left(1+\delta_{2}\right)\right\}$,

$$
\limsup _{n \rightarrow \infty} \frac{\widehat{\gamma}_{n}^{H}\left(k_{0}, k\right)}{a\left(Y_{n-k_{0}, n}\right) /\left[U\left(Y_{n-k_{0}, n}\right)-U\left(Y_{n-k, n}\right)\right]} \leq \varepsilon+(1+\varepsilon) \frac{\frac{\varepsilon^{-1}}{\varepsilon^{-1}-1}-1}{\varepsilon}
$$

almost surely. This, together with a similar lower bound, gives

$$
\lim _{n \rightarrow \infty} \frac{\widehat{\gamma}_{n}^{H}\left(k_{0}, k\right)}{a\left(Y_{n-k_{0}, n}\right) /\left[U\left(Y_{n-k_{0}, n}\right)-U\left(Y_{n-k, n}\right)\right]}=1
$$

almost surely. Replacing $x$ and $t$ in Eq. 4.2 by $Y_{n-k, n} / Y_{n-k_{0}, n}$ and $Y_{n-k_{0}, n}$, respectively, we have

$$
\frac{U\left(Y_{n-k, n}\right)-U\left(Y_{n-k_{0}, n}\right)}{a\left(Y_{n-k_{0}, n}\right)} \stackrel{\text { a.s. }}{<} \frac{(1-\varepsilon)^{-1}\left(Y_{n-k, n} / Y_{n-k_{0}, n}\right)^{\gamma-\varepsilon}-1}{\gamma}(1+\varepsilon)
$$

for all sufficiently large $n$. Lemma 4.2 shows that $Y_{n-k, n} / Y_{n-k_{0}, n} \rightarrow 0$ almost surely. So,

$$
\limsup _{n \rightarrow \infty} \frac{U\left(Y_{n-k, n}\right)-U\left(Y_{n-k_{0}, n}\right)}{a\left(Y_{n-k_{0}, n}\right)} \leq-\frac{1}{\gamma}
$$

almost surely. This, together with a similar lower bound, gives

$$
\lim _{n \rightarrow \infty} \frac{U\left(Y_{n-k, n}\right)-U\left(Y_{n-k_{0}, n}\right)}{a\left(Y_{n-k_{0}, n}\right)}=-\frac{1}{\gamma}
$$


almost surely. So, $a\left(Y_{n-k_{0}, n}\right) /\left[U\left(Y_{n-k_{0}, n}\right)-U\left(Y_{n-k, n}\right)\right] \rightarrow \gamma$ almost surely. Similarly, we can prove $B_{3} \rightarrow-\left\{2^{-\gamma}-1\right\} / \gamma$ and $B_{4} \rightarrow 0$ almost surely. So,

$$
\widehat{\gamma}_{n}^{W}(m)=-\frac{1}{\log 2} \log \left(1+B_{3} \cdot B_{4}\right) \rightarrow 0
$$

almost surely. So, $\widehat{\gamma}_{n}^{\operatorname{Invwh}}\left(k_{0}, k\right) \rightarrow \gamma$ almost surely for the case $\gamma>0$.

(2) The case $\gamma=0$. Firstly, by the arguments similar to the case $\gamma>0$, one can check that

$$
\lim _{n \rightarrow \infty} \frac{\widehat{\gamma}_{n}^{H}\left(k_{0}, k\right)}{a\left(Y_{n-k_{0}, n}\right) /\left[U\left(Y_{n-k_{0}, n}\right)-U\left(Y_{n-k, n}\right)\right]}=1
$$

almost surely. Note that $a(t) \in R V_{0}$. For all sufficiently large $n$, replacing $x$ and $t$ in Eq. 4.3 by $Y_{n-k, n} / Y_{n-k_{0}, n}<1$ and $Y_{n-k_{0}, n}$, respectively, we have

$$
\begin{aligned}
\frac{U\left(Y_{n-k_{0}, n}\right)-U\left(Y_{n-k, n}\right)}{a\left(Y_{n-k_{0}, n}\right)} \stackrel{\text { a.s. }}{<}(1+\varepsilon)\left\{\frac{\left(Y_{n-k, n} / Y_{n-k_{0}, n}\right)^{\varepsilon}-1}{\varepsilon}(1-\varepsilon)+\varepsilon\right\} \\
\rightarrow(1+\varepsilon-1 / \varepsilon)(1+\varepsilon)
\end{aligned}
$$

almost surely as $Y_{n-k, n} / Y_{n-k_{0}, n} \rightarrow 0$ almost surely from Lemma 4.2. Letting $\varepsilon \rightarrow 0$, we obtain

$$
\limsup _{n \rightarrow \infty} \frac{U\left(Y_{n-k_{0}, n}\right)-U\left(Y_{n-k, n}\right)}{a\left(Y_{n-k_{0}, n}\right)} \stackrel{\text { a.s. }}{=}-\infty,
$$

i.e. $a\left(Y_{n-k_{0}, n}\right) /\left[U\left(Y_{n-k_{0}, n}\right)-U\left(Y_{n-k, n}\right)\right] \rightarrow 0$ almost surely. Combining with Eq. 4.4, we obtain $\widehat{\gamma}_{n}^{H}\left(k_{0}, k\right) \rightarrow 0$ almost surely.

Finally, by Lemma 4.2 , we can check that $B_{3} \rightarrow \log 2$ and $B_{4} \rightarrow 0$ almost surely. So,

$$
\widehat{\gamma}_{n}^{W}(m)=-\frac{1}{\log 2} \log \left(1+B_{3} \cdot B_{4}\right) \rightarrow 0
$$

almost surely. So, the strong consistency of $\widehat{\gamma}_{n}^{\text {Invwh }}\left(k_{0}, k\right)$ is proved for $\gamma=0$.

(3) The case $\gamma<0$.

Arguments similar to those of the case $\gamma>0$.

By Lemmas 4.1 and 4.3 , we can obtain

$$
\lim _{n \rightarrow \infty} \frac{\widehat{\gamma}_{n}^{H}\left(k_{0}, k\right)}{a\left(Y_{n-k_{0}, n}\right) /\left[U\left(Y_{n-k_{0}, n}\right)-U\left(Y_{n-k, n}\right)\right]}=\frac{1}{1-\gamma}
$$

almost surely. 
Note that $a(t) \sim-\gamma(U(\infty)-U(t)) \in R V_{\gamma}$ as $\gamma<0$. We can check that Eq. 4.2 also holds as $\gamma<0$. By the arguments similar to the case $\gamma=0$, we can prove that $a\left(Y_{n-k_{0}, n}\right) /\left[U\left(Y_{n-k_{0}, n}\right)-U\left(Y_{n-k, n}\right)\right] \rightarrow 0$ almost surely. Combining with Eq. 4.5, we have $\widehat{\gamma}_{n}^{H}\left(k_{0}, k\right) \rightarrow 0$ almost surely. By Lemma 4.2, one has $B_{3} \rightarrow-\left\{2^{-\gamma}-1\right\} / \gamma$ and $B_{4} \rightarrow-\gamma$ almost surely. So,

$$
\widehat{\gamma}_{n}^{W}(m)=-\frac{1}{\log 2} \log \left(1+B_{3} \cdot B_{4}\right) \rightarrow \gamma
$$

almost surely. The proof of Theorem 2.2 is complete.

Proof of Theorem 2.3 The special properties of the standard Pareto random variables $\left\{Y_{i}, 1 \leq i \leq n\right\}$ can be used to derive asymptotic representations for $B_{s}, s=1,2,3,4$ in Eq. 4.1 under condition Eq. 2.3.

$$
\begin{aligned}
B_{1}= & D_{\gamma}\left(Y_{n-i, n} / Y_{n-k_{0}, n}\right)+k_{\gamma, \rho}\left(Y_{n-i, n} / Y_{n-k_{0}, n}\right) \\
& \cdot A\left(Y_{n-k_{0}, n}\right) \cdot\left(1+o_{P}(1)\right) \\
\stackrel{d}{=} & D_{\gamma}\left(Y_{k_{0}-i, k_{0}}\right)+k_{\gamma, \rho}\left(Y_{k_{0}-i, k_{0}}\right) \cdot A\left(n / k_{0}\right) \cdot\left(1+o_{P}(1)\right) .
\end{aligned}
$$

So, according to whether $\gamma, \rho$ or $\gamma+\rho$ is zero or not, we have the following five cases:

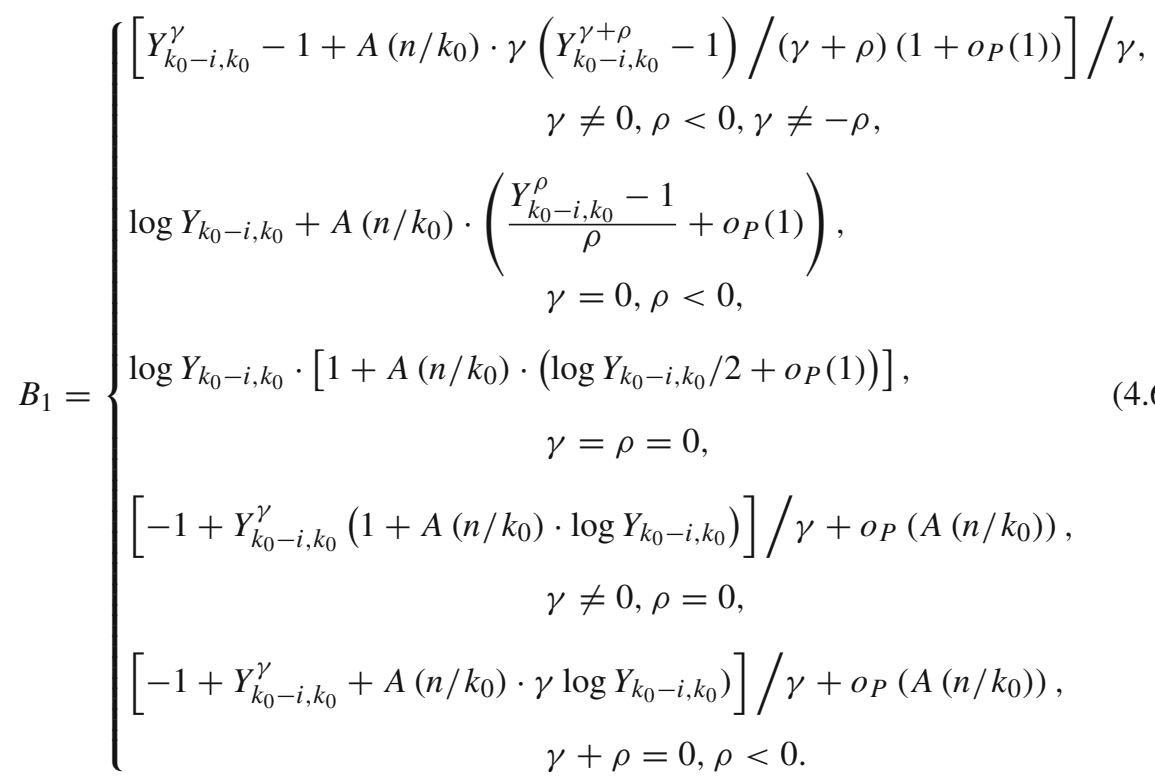


Following the same technique, one can obtain the following representations for $B_{3}$ :

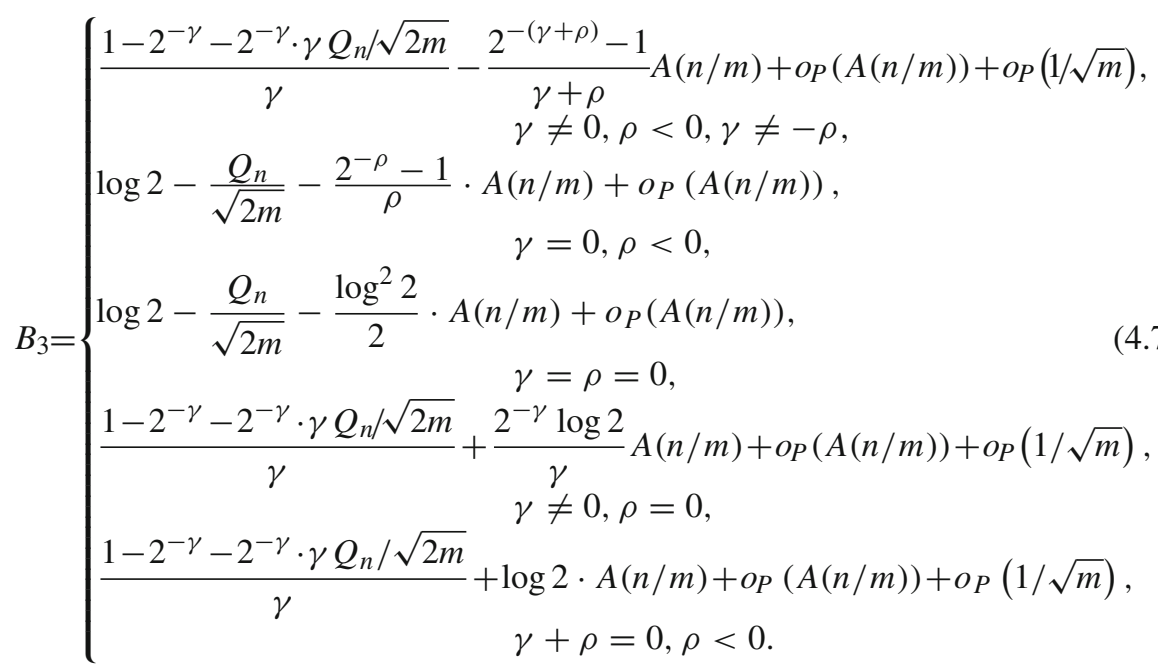

Similarly, one can obtain the following representations for $B_{2}, B_{4}$ under conditions Eqs. 2.2 and 2.5:

$$
\begin{aligned}
B_{2} & =-\frac{a\left(Y_{n-k_{0}, n}\right)}{U\left(Y_{n-k, n}\right)-U\left(Y_{n-k_{0}, n}\right)} \\
& \stackrel{d}{=}-\frac{1}{D_{\gamma}\left(Y_{k-k_{0}, k}^{-1}\right)+k_{\gamma, \rho}\left(Y_{k-k_{0}, k}^{-1}\right) \cdot A\left(Y_{n-k_{0}, n}\right) \cdot\left(1+o_{P}(1)\right)} \\
& = \begin{cases}\gamma\left(1+Y_{k-k_{0}, k}^{-\gamma}-\frac{\gamma}{\gamma+\rho} \cdot A\left(n / k_{0}\right) \cdot I_{\{\gamma+\rho \neq 0, \rho<0\}}\right)\left(1+o_{P}(1)\right), & \text { for } \gamma>0, \\
\frac{1}{\log Y_{k-k_{0}, k}\left(1+o_{P}(1)\right),} & \text { for } \gamma=0, \\
-\gamma Y_{k-k_{0}, k}^{\gamma}\left(1+o_{P}(1)\right), & \text { for } \gamma<0\end{cases}
\end{aligned}
$$

and

$$
\begin{aligned}
B_{4} & =\frac{a\left(Y_{n-m+1, n}\right)}{U\left(Y_{n, n}\right)-U\left(Y_{n-m+1, n}\right)} \\
& =\frac{1}{D_{\gamma}\left(m S_{n}\right)+k_{\gamma, \rho}\left(m S_{n}\right) \cdot A(n / m) \cdot\left(1+o_{P}(1)\right)} \\
& = \begin{cases}\gamma\left(m S_{n}\right)^{-\gamma}\left(1+o_{P}(1)\right), & \text { for } \gamma>0, \\
\frac{1}{\log m}\left(1-\frac{\log S_{n}}{\log m}\right)\left(1+o_{P}(1)\right), & \text { for } \gamma=0, \\
-\gamma\left[1+\left(m S_{n}\right)^{\gamma}-\frac{\gamma}{\gamma+\rho} A(n / m) \cdot I_{\{\rho<0\}}\right]\left(1+o_{P}(1)\right), & \text { for } \gamma<0 .\end{cases}
\end{aligned}
$$


Combining Eqs. 4.6, 4.7, 4.8 and 4.9, we can establish asymptotic representations for $\widehat{\gamma}_{n}^{\text {Invwh }}\left(k_{0}, k\right)$ for the cases $\gamma>0, \gamma=0$ and $\gamma<0$.

(1) The case $\gamma>0$.

Consider the case $\rho<0$ and $\rho \neq-\gamma$. Combining Eqs. 4.6 and 4.8, we have

$$
\begin{aligned}
& \frac{1}{k_{0}} \sum_{i=0}^{k_{0}-1} \log \left(1+B_{1} \cdot B_{2}\right)=\gamma \cdot \frac{1}{k_{0}} \sum_{i=0}^{k_{0}-1} \log Y_{k_{0}-i, k_{0}}+A\left(n / k_{0}\right) \\
& \cdot \frac{1}{k_{0}} \sum_{i=0}^{k_{0}-1} \gamma\left(Y_{k_{0}-i, k_{0}}^{\rho}-1\right) /(\gamma+\rho) \\
& +Y_{k-k_{0}, k}^{-\gamma} \frac{1}{k_{0}} \sum_{i=0}^{k_{0}-1}\left(1-Y_{k_{0}-i, k_{0}}^{-\gamma}\right) \\
& +o_{P}\left(A\left(n / k_{0}\right)\right)+o_{P}\left(\left(k_{0} / k\right)^{\gamma}\right) \\
& =\gamma+\frac{\gamma P_{n}}{\sqrt{k}_{0}}+\frac{\gamma\left[E Y^{\rho}-1\right]}{\gamma+\rho} \cdot A\left(n / k_{0}\right) \\
& +E\left(1-Y^{-\gamma}\right) \cdot Y_{k-k_{0}, k}^{-\gamma}+o_{P}\left(\frac{1}{\sqrt{k_{0}}}\right) \\
& +o_{P}\left(A\left(n / k_{0}\right)\right)+o_{P}\left(\left(k_{0} / k\right)^{\gamma}\right) \\
& =\gamma+\frac{\gamma P_{n}}{\sqrt{k_{0}}}+\frac{\gamma \rho}{(1-\rho)(\gamma+\rho)} \cdot A\left(n / k_{0}\right) \\
& +\frac{\gamma}{1+\gamma} \cdot Y_{k-k_{0}, k}^{-\gamma}+o_{P}\left(\frac{1}{\sqrt{k_{0}}}\right) \\
& +o_{P}\left(A\left(n / k_{0}\right)\right)+o_{P}\left(\left(k_{0} / k\right)^{\gamma}\right) \text {. }
\end{aligned}
$$

If $\rho=0$, or $\rho<0$ and $\rho=-\gamma$ then one can easily derive the asymptotic representation. So,

$$
\begin{aligned}
& \frac{1}{k_{0}} \sum_{i=0}^{k_{0}-1} \log \left(1+B_{1} \cdot B_{2}\right) \\
& =\gamma+\frac{\gamma P_{n}}{\sqrt{k_{0}}}+\left\{\frac{\gamma}{(1-\rho)(1+\gamma)} \cdot I_{\{\rho<0, \rho \neq-\gamma\}}+\frac{\gamma}{(1+\gamma)^{2}} \cdot I_{\{\rho<0, \rho=-\gamma\}}+I_{\{\rho=0\}}\right\} \\
& \quad \cdot A\left(\frac{n}{k_{0}}\right)+\frac{\gamma}{1+\gamma} Y_{k-k_{0}, k}^{-\gamma}+o_{P}\left(\frac{1}{\sqrt{k_{0}}}\right)+o_{P}\left(A\left(\frac{n}{k_{0}}\right)\right)+o_{P}\left(\left(k_{0} / k\right)^{\gamma}\right) .
\end{aligned}
$$


Also,

$$
\frac{1}{\log 2} \log \left(1+B_{3} \cdot B_{4}\right)=\frac{1-2^{-\gamma}}{\log 2}\left(m S_{n}\right)^{-\gamma}+o_{P}\left(m^{-\gamma}\right)
$$

So, if we sum the above two parts, we prove Eq. 2.6.

(2) The case $\gamma=0$. One can easily prove Eq. 2.7 by use of the same method.

If $\rho=0$ then

$$
\begin{aligned}
& \frac{1}{k_{0}} \sum_{i=0}^{k_{0}-1} \log (1+\left.B_{1} \cdot B_{2}\right) \\
&=\frac{1}{k_{0}} \sum_{i=0}^{k_{0}-1} \log \{1 \frac{\log Y_{k_{0}-i, k_{0}}}{\log Y_{k-k_{0}, k}} \\
&\left.\times\left[1+A\left(\frac{n}{k_{0}}\right) \frac{\log Y_{k_{0}-i, k_{0}}}{2}+o_{P}\left(A\left(\frac{n}{k_{0}}\right)\right)\right]\left(1+o_{P}(1)\right)\right\} \\
&=\frac{1}{\log Y_{k-k_{0}, k}}\left\{\frac{1}{k_{0}} \sum_{i=0}^{k_{0}-1} \log Y_{k_{0}-i, k_{0}}+A\left(\frac{n}{k_{0}}\right) \frac{1}{k_{0}} \sum_{i=0}^{k_{0}-1} \frac{\left.\log ^{2} Y_{k_{0}-i, k_{0}}+o_{P}\left(A\left(\frac{n}{k_{0}}\right)\right)\right\}}{2}\left\{1+\frac{P_{n}}{\sqrt{k_{0}}}+A\left(\frac{n}{k_{0}}\right)+o_{P}\left(A\left(\frac{n}{k_{0}}\right)\right)+o_{P}\left(\frac{1}{\sqrt{k_{0}}}\right)\right\} .\right.
\end{aligned}
$$

If $\rho<0$ then

$$
\begin{aligned}
& \frac{1}{k_{0}} \sum_{i=0}^{k_{0}-1} \log \left(1+B_{1} \cdot B_{2}\right) \\
& \quad=\frac{1}{\log Y_{k-k_{0}, k}}\left[1+\frac{P_{n}}{\sqrt{k_{0}}}+\frac{1}{1-\rho} \cdot A\left(\frac{n}{k_{0}}\right)+o_{P}\left(A\left(\frac{n}{k_{0}}\right)\right)+o_{P}\left(\frac{1}{\sqrt{k_{0}}}\right)\right] .
\end{aligned}
$$

Also,

$$
\frac{1}{\log 2} \log \left(1+B_{3} \cdot B_{4}\right)=\frac{1}{\log m} \cdot\left(1-\frac{\log S_{n}}{\log m}+O_{P}\left(A\left(\frac{n}{m}\right)\right)+O_{P}\left(\frac{1}{\sqrt{m}}\right)\right) .
$$

(3) The case $\gamma<0$. One can obtain

$$
\begin{aligned}
\frac{1}{k_{0}} \sum_{i=0}^{k_{0}-1} \log \left(1+B_{1} \cdot B_{2}\right)= & -Y_{k-k_{0}, k}^{\gamma}\left\{\frac{\gamma}{1-\gamma}+\left[\frac{\gamma}{1-\rho-\gamma} I_{\{\rho<0\}}+\frac{1}{(1-\gamma)^{2}}\right.\right. \\
& \left.\left.\times I_{\{\rho=0\}}\right] A\left(\frac{n}{k_{0}}\right)+o_{P}\left(\frac{1}{\sqrt{k_{0}}}\right)+o_{P}\left(A\left(\frac{n}{k_{0}}\right)\right)\right\} .
\end{aligned}
$$


Also,

$$
\begin{aligned}
\frac{1}{\log 2} \log \left(1+B_{3} \cdot B_{4}\right)= & \frac{\left(1-2^{\gamma}\right)\left(m S_{n}\right)^{\gamma}}{\log 2}-\gamma+\frac{\gamma Q_{n}}{\sqrt{2 m} \log 2} \\
& +\left\{\frac{\gamma\left(2^{-\rho}-1\right)}{(\gamma+\rho) \log 2} I_{\{\rho<0\}}-I_{\{\rho=0\}}\right\} A\left(\frac{n}{m}\right) \\
& +o_{P}\left(m^{\min (-1 / 2, \gamma)}\right) .
\end{aligned}
$$

Combining the above results, the asymptotic distributional representations in Eqs. 2.6, 2.7 and 2.8 follow. The proof of Theorem 2.3 is complete.

Proof of Theorem 2.4 Firstly, we need the following results:

$$
\begin{aligned}
& \sqrt{k_{0}} A\left(n / k_{0}\right) \rightarrow \lambda \Longrightarrow A\left(\frac{n}{k_{0}}\right)=O\left(\frac{1}{\sqrt{k_{0}}}\right) \ll \frac{1}{\log k_{0}}, \\
& k_{0}^{\min (-\gamma, 1 / 2)} A\left(n / k_{0}\right) \rightarrow \lambda \Longrightarrow A\left(\frac{n}{k_{0}}\right)=O\left(\frac{1}{k_{0}^{\min (-\gamma, 1 / 2)}}\right) \ll \frac{1}{\log k_{0}}, \gamma<0, \\
& \frac{\sqrt{k_{0}}}{\log \left(k_{0} / k\right)} \rightarrow 0 \Longrightarrow\left(\frac{k_{0}}{k}\right)^{\gamma} \ll \frac{1}{\sqrt{k}_{0}}, \gamma>0, \\
& \frac{\sqrt{k_{0}}}{\log \left(k_{0} / k\right)} \rightarrow 0 \Longrightarrow \frac{1}{\log \left(k_{0} / k\right)} \ll \frac{1}{\log ^{2} k_{0}} \ll \frac{1}{\log k_{0}}
\end{aligned}
$$

and

$$
\frac{k_{0}^{\min (1 / 2,-\gamma)}}{\log \left(k_{0} / k\right)} \rightarrow 0 \Longrightarrow\left(\frac{k_{0}}{k}\right)^{-\gamma} \ll \frac{1}{k_{0}^{\min (1 / 2,-\gamma)}}, \gamma<0 .
$$

So, for $\gamma>0$, according to Eqs. 2.6, 4.10 and 4.12,

$$
\begin{aligned}
\widehat{\gamma}_{n}^{\text {Invwh }}\left(k_{0}, k\right)= & \gamma+\frac{\gamma P_{n}}{\sqrt{k_{0}}}+\frac{\left(2^{\gamma}-1\right)\left(-S_{n}^{-\gamma}\right)}{\log 2 \cdot k_{0}^{\gamma}}+o_{P}\left(\frac{1}{\sqrt{k_{0}}}\right)+o_{P}\left(k_{0}^{-\gamma}\right) \\
& +A\left(n / k_{0}\right)\left\{\left[\frac{\gamma \rho}{(1-\rho)(\gamma+\rho)} \cdot I_{\{\gamma \neq-\rho\}}+\frac{\gamma}{(1+\gamma)^{2}} \cdot I_{\{\gamma=-\rho\}}\right]\right. \\
& \left.\cdot I_{\{\rho<0\}}+I_{\{\rho=0\}}\right\} .
\end{aligned}
$$


For $\gamma=0$, according to Eqs. 2.7 and 4.13,

$$
\begin{aligned}
\widehat{\gamma}_{n}^{\text {Invwh }}\left(k_{0}, k\right)= & -\frac{1}{\log m}+\frac{\log S_{n}}{\log ^{2} m}+o_{P}\left(\frac{1}{\log ^{2} m}\right) \\
= & -\frac{1}{\log \left(k_{0} / 2\right)}+\frac{\log S_{n}}{\left(\log ^{2} k_{0}\right)(1+o(1))}+\frac{\log \left(1+1 / k_{0}\right)}{\left(\log ^{2} k_{0}\right)(1+o(1))} \\
& +o_{P}\left(\frac{1}{\log ^{2} k_{0}}\right)
\end{aligned}
$$

since $m=\left[\left(k_{0}+1\right) / 2\right]$. For $\gamma<0$, according to Eqs. 2.8, 4.11 and 4.14,

$$
\begin{aligned}
\widehat{\gamma}_{n}^{\text {Invwh }}\left(k_{0}, k\right)= & \gamma+\frac{\left(2^{-\gamma}-1\right)\left(-S_{n}^{\gamma}\right)}{\log 2 \cdot k_{0}^{-\gamma}}-\frac{\gamma Q_{n}}{\sqrt{k_{0}} \log 2}+o_{P}\left(k_{0}^{\min (-1 / 2, \gamma)}\right) \\
& +A\left(n / k_{0}\right)\left\{\frac{\gamma\left(2^{\rho}-1\right)}{(\gamma+\rho) \log 2} \cdot I_{\{\rho<0\}}+2^{\rho} \cdot I_{\{\rho=0\}}\right\} .
\end{aligned}
$$

Noting that $Q_{n} \stackrel{d}{\rightarrow} Q \sim N(0,1), S_{n} \stackrel{d}{\rightarrow} S$ with the d.f $\exp (-1 / y), y>0, S$ and $Q$ are independent (see Eq. 2.4), $P_{n} \stackrel{d}{\rightarrow} P \sim N(0,1)$, and $P_{n}$ and $S_{n}$ are asymptotically independent (cf. Corollary 2.1 of Fraga Alves 2001b), we obtain the desired results. The proof is complete.

Proof of Theorem 2.5 We only consider the case $|\gamma|<1 / 2$. The arguments for the other cases are similar and so the details are not given.

According to Theorem 2.3, we have

$$
\begin{aligned}
\widehat{\gamma}_{n}^{\text {Invwh }}\left(k_{0}, k\right)= & \gamma+\frac{\sigma(\gamma)}{\sqrt{k_{0}}}\left\{P_{n} I_{\{\gamma>0\}}-Q_{n} I_{\{\gamma<0\}}\right\}+c_{1}\left(\frac{k_{0}}{k}\right)^{|\gamma|}+c_{2} c\left(\frac{k_{0}}{n}\right)^{|\rho|} \\
& +o_{P}\left(\left(\frac{k_{0}}{k}\right)^{|\gamma|}\right)+o_{P}\left(\left(\frac{k_{0}}{n}\right)^{|\rho|}\right)+o_{P}\left(1 / \sqrt{k_{0}}\right) .
\end{aligned}
$$

For $|\gamma|<1 / 2$,

$$
\begin{aligned}
\widehat{\gamma}_{n}^{\text {Invwh }}\left(k_{0}, k\right)= & \gamma-\frac{\sigma(\gamma)}{k_{0}^{|\gamma|}} \cdot \frac{S_{n}^{-|\gamma|}}{\sqrt{\Gamma(1+2|\gamma|)-\Gamma^{2}(1+|\gamma|)}} c_{1}\left(\frac{k_{0}}{k}\right)^{|\gamma|}+c_{2} c\left(\frac{k_{0}}{n}\right)^{|\rho|} \\
& +o_{P}\left(\left(\frac{k_{0}}{k}\right)^{|\gamma|}\right)+o_{P}\left(\left(\frac{k_{0}}{n}\right)^{|\rho|}\right)+o_{P}\left(1 / k_{0}^{|\gamma|}\right) \\
= & \gamma-\frac{\sigma(\gamma)}{k_{0}^{|\gamma|}} \cdot \frac{S_{n}^{-|\gamma|}+\Gamma(1+|\gamma|)}{\sqrt{\Gamma(1+2|\gamma|)-\Gamma^{2}(1+|\gamma|)}} \frac{c_{3}}{k_{0}^{|\gamma|}}+c_{1}\left(\frac{k_{0}}{k}\right)^{|\gamma|} \\
& +c_{2} c\left(\frac{k_{0}}{n}\right)^{|\rho|}+o_{P}\left(\left(\frac{k_{0}}{k}\right)^{|\gamma|}\right)+o_{P}\left(\left(\frac{k_{0}}{n}\right)^{|\rho|}\right)+o_{P}\left(1 / k_{0}^{|\gamma|}\right),
\end{aligned}
$$


where

$$
c_{3}=\frac{-\sigma(\gamma) \cdot \Gamma(1+|\gamma|)}{\sqrt{\Gamma(1+2|\gamma|)-\Gamma^{2}(1+|\gamma|)}}<0
$$

Firstly, we discuss the asymptotic mean squared error of $\widehat{\gamma}_{n}^{\text {Invwh }}\left(k_{0}, k\right)$. If $|\gamma| \leq$ $|\rho|$ then $\left(k_{0} / n\right)^{|\rho|} \ll\left(k_{0} / k\right)^{|\gamma|}$, which implies

$$
\operatorname{MSE} E_{\infty}\left(\widehat{\gamma}_{n}^{\text {Invwh }}\left(k_{0}, k\right)\right)=\frac{\sigma^{2}(\gamma)}{k_{0}^{2|\gamma|}}+c_{1}^{2}\left(\frac{k_{0}}{k}\right)^{2|\gamma|}+\frac{c_{3}^{2}}{k_{0}^{2|\gamma|}} .
$$

The optimal sample fraction is $k_{0}^{(o p t)}=k_{0}^{(11)}$. Under this optimal sequence, the asymptotical bias is $\left(c_{3}+\sqrt{c_{3}^{2}+\sigma^{2}(\gamma)}\right) /\left(k_{0}^{11}\right)^{|\gamma|}$. If $|\gamma|>|\rho|$, we can obtain

$$
M S E_{\infty}\left(\widehat{\gamma}_{n}^{\text {Invwh }}\left(k_{0}, k\right)\right)=\frac{\sigma^{2}(\gamma)}{k_{0}^{2|\gamma|}}+\left[c_{1}\left(\frac{k_{0}}{k}\right)^{|\gamma|}+c_{2} c\left(k_{0} / n\right)^{|\rho|}+c_{3} / k_{0}^{|\gamma|}\right]^{2} .
$$

We must consider the relative weights of the above representation. There are three cases:

- Suppose $k^{1 / 2} \ll n^{|\rho| /(|\rho|+|\gamma|)}$. We have

$$
k^{|\gamma| /(|\gamma|+\rho)} n^{\rho /(|\gamma|+\rho)} \ll k^{1 / 2} \ll n^{|\rho| /(|\rho|+|\gamma|)} .
$$

If $k_{0} \ll k^{1 / 2}\left(\ll n^{|\rho| /(|\rho|+|\gamma|)}\right)$ then

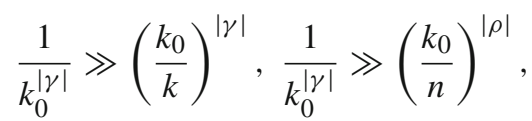

which implies $\operatorname{MSE} E_{\infty}\left(\widehat{\gamma}_{n}^{\text {Invwh }}\left(k_{0}, k\right)\right)=\left\{\sigma^{2}(\gamma)+c_{3}^{2}\right\} / k_{0}^{2|\gamma|}$ is a decreasing function on $k_{0}$. On the other hand, if $k_{0} \gg k^{1 / 2}\left(\gg k^{|\rho| /(\rho+|\gamma|)} \cdot n^{\rho /(|\gamma|+\rho)}\right.$ then

$$
\frac{1}{k_{0}^{|\gamma|}} \ll\left(\frac{k_{0}}{k}\right)^{|\gamma|},\left(\frac{k_{0}}{n}\right)^{|\rho|} \ll\left(\frac{k_{0}}{k}\right)^{|\gamma|},
$$

and $\operatorname{MSE} E_{\infty}\left(\widehat{\gamma}_{n}^{\text {Invwh }}\left(k_{0}, k\right)\right)=c_{1}^{2}\left(k_{0} / k\right)^{2|\gamma|}$ is an increasing function on $k_{0}$. In order to balance the bias and the variance of the estimator, $k_{0}^{(o p t)}$ must be the same order of $k^{1 / 2}$. Moreover, $\operatorname{MSE} E_{\infty}\left(\widehat{\gamma}_{n}^{\text {Invwh }}\left(k_{0}, k\right)\right)=\left\{\sigma^{2}(\gamma)+c_{3}^{2}\right\} / k_{0}^{2|\gamma|}+$ $c_{1}^{2}\left(k_{0} / k\right)^{2|\gamma|}$, which implies that the optimal sample fraction is $k_{0}^{(o p t)}=k_{0}^{(11)}$.

- Suppose $k^{1 / 2} \gg n^{|\rho| /(|\rho|+|\gamma|)}$. We have

$$
k^{|\gamma| /(|\gamma|+\rho)} \cdot n^{\rho /(|\gamma|+\rho)} \gg k^{1 / 2} \gg n^{|\rho| /(|\rho|+|\gamma|)} .
$$


If $k_{0} \ll k^{|\rho| /(|\rho|+|\gamma|)}\left(\ll k^{1 / 2}\right)$ then

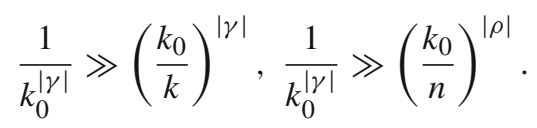

So, $M S E_{\infty}\left(\widehat{\gamma}_{n}^{\text {Invwh }}\left(k_{0}, k\right)\right)=\left\{\sigma^{2}(\gamma)+c_{3}^{2}\right\} / k_{0}^{2|\gamma|}$ is a decreasing function on $k_{0}$. On the other hand, if $k_{0} \gg k^{|\rho| /(|\rho|+|\gamma|)}$ then $1 / k_{0}^{|\gamma|} \ll\left(k_{0} / n\right)^{|\rho|}$. In order to balance the bias and the variance of the estimator, we take $k_{0}$ as the order of $k^{|\rho| /(|\rho|+|\gamma|)}$. Moreover,

$$
\frac{1}{k_{0}^{|\gamma|}}=O\left(\left(\frac{k_{0}}{n}\right)^{|\rho|}\right) \gg\left(\frac{k_{0}}{k}\right)^{|\gamma|}
$$

and $\operatorname{MSE} E_{\infty}\left(\widehat{\gamma}_{n}^{\text {Invwh }}\left(k_{0}, k\right)\right)=\left\{\sigma^{2}(\gamma)+c_{3}^{2}\right\} / k_{0}^{2|\gamma|}+\left(c_{2} c\right)^{2}\left(k_{0} / n\right)^{2|\rho|}$. This yields the optimal sample fraction $k_{0}^{(o p t)}=k_{0}^{(21)}$.

Now if $k_{0}$ is any sequence such that $n^{|\rho| /(|\rho|+|\gamma|)} \ll k_{0} \ll k^{|\gamma| /(|\gamma|+\rho)} \cdot n^{\rho /(|\gamma|+\rho)}$ then

$$
\frac{1}{k_{0}^{|\gamma|}} \ll\left(\frac{k_{0}}{n}\right)^{|\rho|},\left(\frac{k_{0}}{k}\right)^{|\gamma|} \ll\left(\frac{k_{0}}{n}\right)^{|\rho|} .
$$

So,

$$
\operatorname{MSE} E_{\infty}\left(\widehat{\gamma}_{n}^{\text {Invwh }}\left(k_{0}, k\right)\right)=\left[c_{1}\left(\frac{k_{0}}{k}\right)^{|\gamma|}+c_{2} c\left(\frac{k_{0}}{n}\right)^{|\rho|}+c_{3} / k_{0}^{|\gamma|}\right]^{2} .
$$

Note that $c_{1}>0, c_{2} \gamma>0$ and $c_{3}<0$, so the sign of the function $A(t)$ is critical to the solution to the optimization problem.

When $c \gamma>0$ (which implies $\left.c c_{2}>0\right), \operatorname{MSE} E_{\infty}\left(\widehat{\gamma}_{n}^{\text {Invwh }}\left(k_{0}, k\right)\right)=\left[c_{3} / k_{0}^{|\gamma|}+\right.$ $\left.\left(c_{2} c\right)\left(k_{0} / n\right)^{|\rho|}\right]^{2}$. Define $k_{0}^{(o p t)}=k_{0}^{(31)}$, the solution to the optimization problem such that $M S E_{\infty}\left(\widehat{\gamma}_{n}^{\operatorname{Invwh}}\left(k_{0}, k\right)\right)=0$.

When $c \gamma<0$ (which implies $c c_{2}<0$ ), define the sequence $k_{0}^{(3)}$ of the same order of $k^{|\gamma| /(|\gamma|+\rho)} \cdot n^{\rho /(|\gamma|+\rho)}$ in such a way that $\operatorname{MSE} E_{\infty}\left(\widehat{\gamma}_{n}^{\operatorname{Invwh}}\left(k_{0}, k\right)\right)=0$.

- Suppose $k \sim D \cdot n^{2|\rho| /(|\gamma|+|\rho|)}$. Then

$$
k^{|\gamma| /(|\gamma|+\rho)} \cdot n^{\rho /(|\gamma|+\rho)} \sim k^{1 / 2} \sim n^{|\rho| /(|\rho|+|\gamma|)},
$$

i.e.

$$
\frac{1}{k_{0}^{|\gamma|}} \sim\left(\frac{k_{0}}{k}\right)^{|\gamma|} \sim\left(\frac{k_{0}}{n}\right)^{|\rho|} .
$$

Putting $k_{0}^{(o p t)}=D_{1} \cdot n^{|\rho| /(|\gamma|+|\rho|)}$, we have

$$
\operatorname{MSE} E_{\infty}\left(\widehat{\gamma}_{n}^{\text {Invwh }}\left(k_{0}^{\text {opt }}, k\right)\right)=g_{\gamma, \rho, c, D}\left(D_{1}\right) \cdot n^{-2|\rho||\gamma| /(|\gamma|+|\rho|)},
$$


where

$$
g_{\gamma, \rho, c, D}\left(D_{1}\right)=\left\{\frac{\sigma^{2}(\gamma)}{D_{1}^{2|\gamma|}}+\left[c_{1}\left(\frac{D_{1}}{D}\right)^{|\gamma|}+c_{2} c D_{1}^{|\rho|}+c_{3} / D_{1}^{|\gamma|}\right]^{2}\right\} .
$$

The function $g_{\gamma, \rho, c, D}\left(D_{1}\right)$ is minimal for $D_{1}=D_{1}(\gamma, \rho, c, D)$ which satisfies the equation:

$$
\begin{aligned}
\frac{c_{1}^{2}}{D^{2|\gamma|}} & \cdot D_{1}^{4|\gamma|}+\frac{c_{1} c_{2} c(1+|\rho / \gamma|)}{D^{|\gamma|}} \cdot D_{1}^{3|\gamma|+|\rho|}+|\rho / \gamma|\left(c_{2} c\right)^{2} \\
& \cdot D_{1}^{2|\gamma|+2|\rho|}-(1-|\rho / \gamma|) \cdot c c_{2} c_{3} \cdot D_{1}^{|\gamma|+|\rho|}=\sigma^{2}(\gamma)+c_{3}^{2} .
\end{aligned}
$$

Acknowledgments The authors would like to thank the Editor-in-Chief, the Associate Editor and the two referees for carefully reading and for their comments which greatly improved the paper.

\section{References}

Araújo Santos, P., Fraga Alves, M.I., Gomes, M.I.: Peaks over random threshold methodology for tail index and quantile estimation. Revstat 4, 227-247 (2006)

Beirlant, J., Vynckier, P., Teugels, J.L.: Excess functions and estimation of the extreme-value index. Bernoulli 2, 293-318 (1996)

Caeiro, F., Gomes, M.I., Pestana, D.D. Direct reduction of bias of the classical Hill estimator. Revstat 3, $113-136(2005)$

de Haan, L.: Extreme value statistics. In: Galambos, J., Lechner, J., Simiu, E. (eds.) Extreme Value Theory and Applications, pp. 93-122. Kluwer Academic Publications, Dordrecht (1994)

de Haan, L., Ferreira, A.: Extreme Value Theory: An Introduction. Springer, New York (2006)

de Haan, L., Peng, L.: Comparison of tail index estimators. Stat. Neerl. 52, 60-70 (1998)

Dekkers, A.L.M., de Haan, L.: On the estimation the extreme-value index and large quantile estimation. Ann. Stat. 17, 1795-1832 (1989)

Dekkers, A.L.M., Einmahl, J.H.J., de Haan, L.: A moment estimator for the index of an extreme-value distribution. Ann. Stat. 17, 1833-1855 (1989)

Draisma, G., de Haan, L., Peng, L., Pereira, T.T.: A bootstrap-based method to achieve optimality in estimating the extreme-value index. Extremes 4, 367-404 (1999)

Falk, M.: Some best parameter estimates for distributions with finite endpoint. Statistics 27, 115-125 (1995)

Fraga Alves, M.I.: A location invariant Hill-type estimator. Extremes 4, 199-217 (2001a)

Fraga Alves, M.I.: Weiss-Hill estimator. Test 10, 203-224 (2001b)

Fraga Alves, M.I., Gomes M.I., de Haan, L., Neves, C.: Mixed moment estimators and location invariant alternatives. Extremes 12, 149-185 (2009)

Gomes, M.I., Henriques Rodrigues, L.: Tail index estimation for heavy tails: accommodation of bias in the excesses over a high threshold. Extremes 11, 303-328 (2008)

Gomes, M.I., Martins, M.J.: "Asymptotically unbiased" estimators of the tail index based on external estimation of the second order parameter. Extremes 5, 5-31 (2002)

Gomes, M.I., Martins, M.J.: Bias reduction and explicit estimation of the tail index. J. Stat. Plan. Inference 124, 361-378 (2004)

Gomes, M.I., Pestana, D.: A sturdy reduced bias extreme quantile (VaR) estimator. J. Am. Stat. Assoc. 102, 280-292 (2007) 
Gomes, M.I, de Haan, L., Henriques Rodrigues, L.: Tail index estimation for heavy-tailed models: accommodation of bias in weighted log-excesses. J. R. Stat. Soc., Ser. B 70, 31-53 (2008a)

Gomes, M.I., Fraga Alves, M.I., Araújo Santos, P.: PORT Hill and moment estimators for heavy-tailed models. Commun. Stat., Simul. Comput. 37, 1281-1306 (2008b)

He, L.: Convergence of a kind of Pickands-type estimator. Acta Math. Sin., Chinese Series 47, 805-810 (2004)

Hill, B.: A simple general approach to inference about the tail of a distribution. Ann. Stat. 3, 1163-1174 (1975)

Li, J., Peng, Z., Nadarajah, S.: A class of unbiased location invariant Hill-type estimators for heavy tailed distributions. Electronic Journal of Statistics 2, 829-847 (2008)

Li, J., Peng, Z., Nadarajah, S.: Asymptotic normality of location invariant heavy tail index estimator. Extremes 13, 269-290 (2010)

Ling, C., Peng, Z., Nadarajah, S.: A location invariant Moment-type estimator I. Theory Probab. Math. Stat. 76, 23-31 (2007a)

Ling, C., Peng, Z., Nadarajah, S.: A location invariant Moment-type estimator II. Theory Probab. Math. Stat. 77, 177-189 (2007b)

Ling, C., Peng, Z., Nadarajah, S.: Selecting the optimal sample fraction in location invariant moment-type estimators. Theory Probab. Appl. (2009, in press)

Müller, S., Hüsler, J.: Iterative estimation of the extreme value index. Methodology and Computing in Applied Probability 2, 139-145 (2005)

Peng, Z.: Extension of Pickands' estimator. Acta Math. Appl. Sin., Chinese Series 40, 759-762 (1997)

Peng, L.: Asymptotically unbiased estimator for the extreme-value index. Statistics and Probability Letters 2, 107-115 (1998a)

Peng, Z.: A kind of simplified Pickands-type estimator. Acta Math. Appl. Sin., Chinese Series 4, 539-542 (1998b)

Peng, L., Qi, Y.: A new calibration method of constructing empirical likelihood-based confidence intervals for the tail index. Aust. N. Z. J. Stat. 48, 59-66 (2006a)

Peng, L., Qi, Y.: Confidence regions for high quantiles of a heavy tailed distribution. Ann. Stat. 4, 19641986 (2006b)

Peng, Z., Nadarajah, S.: The Pickands' estimator of the negative extreme value index. Acta Scientiarum Naturalium Universitatis Pekinensis 37, 12-19 (2001)

Pickands, J.: Statistical inference using extreme order statistics. Ann. Stat. 3, 119-131 (1975)

Qi, Y.: On the tail index of a heavy tailed distribution. Ann. Inst. Stat. Math. 62, 277-298 (2010)

Segers, J.: Generalized Pickands estimators for the extreme value index. J. Stat. Plan. Inference 128, 381396 (2005)

Weiss, L.: Asymptotic inference about a density function at the end of its range. Nav. Res. Logist. Q. 1, 111-114 (1971) 